\title{
Towards a definition of the Quantum Ergodic Hierarchy: Kolmogorov and Bernoulli systems
}

\author{
Mario Castagnino $^{1}$ and Ignacio Gomez ${ }^{2}$
}

September 17, 2013

\begin{abstract}
In this paper we translate the two higher levels of the Ergodic Hierarchy 1, the Kolmogorov level and the Bernoulli level, to quantum language. Moreover, this paper can be considered as the second part of 22. As in paper [2, we consider the formalism where the states are positive functionals on the algebra of observables and we use the properties of the Wigner transform [3]. We illustrate the physical relevance of the Quantum Ergodic Hierarchy with two emblematic examples of the literature: the Casati-Prosen model [4, [5] and the kicked rotator [6], [7, [8].
\end{abstract}

\author{
1- Instituto de Física de Rosario (IFIR-CONICET) and \\ Instituto de Astronomía y Física del Espacio, \\ Casilla de Correos 67, Sucursal 28, 1428 Buenos Aires, Argentina. \\ 2- Instituto de Física de Rosario (IFIR-CONICET), Rosario, Argentina
}

Key words: Ergodic-Mixing-Kolmogorov-Bernoulli-EH-QEH

\section{Introduction}

This paper is based on [9] which presents a three theories structure of classical chaos: Ergodic Hierarchy, Lyapunov exponents and Complexity. The first two theories are related to the Pesin's theorem [10] and the last two ones to the Brudno's theorem [9]. The Pesin's theorem expresses the equivalence between the KS entropy and the exponential divergence of trajectories by the presence of positive Lyapunov exponents, and the positivity of these exponents is a necessary and sufficient condition for chaos. On the other hand, the Brudno's theorem expresses the equivalence between the complexity of almost every point of the phase space and the KS entropy. The theoretical relation between these chaos indicators, KS entropy, complexity and Lyapunov exponents are sketched in a "chaos pyramid" in the figure 1. According to this structure Ergodic Hierarchy is one of the features of classical chaos. We have presented in our previous paper 2 a proposal to define the first two steps of a Quantum Ergodic Hierarchy, i.e. Quantum Ergodic and Quantum Mixing systems. Then the only purpose of this paper is to complete the Quantum Ergodic Hierarchy with two more steps: Quantum Kolmogorov and Quantum Bernouilli. On the other hand the large majority of works on Quantum Chaos follows a different line. That is, in books [11], [12], 6], [7], and [8] only "bra" and "kets" appear. Concepts like "observables" 


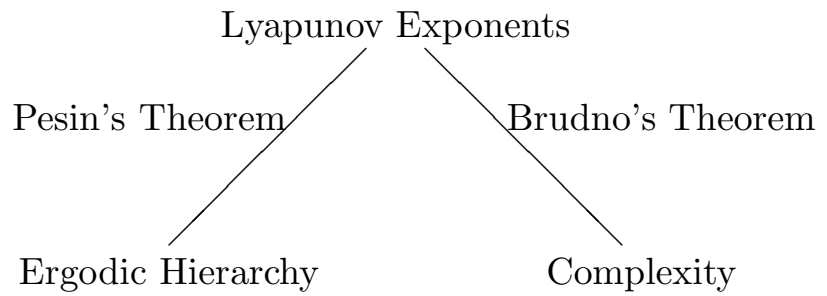

Figure 1: The "chaos pyramid" is a diagram of the relationships beetween the three theories structures: Ergodic Hierarchy, Lyapunov and Complexity through the Pesin and Brudno theorems.

and "functionals" are not taken into account in a fundamental way in these books. Then a crucial definition of the mixing systems does not appear in these books, i.e. as the property of a quantum system with weak limit reaches equilibrium 22. Essentially the way to introduce a Quantum Ergodic Hierarchy cannot be based in the study of quantum closed systems with just "bra" and "kets". Other concepts must be introduced as "observables" and "mixed states".

More precisely, following the Ballentine's book [13], where an axiomatic structure for Quantum Mechanics is sketched, the primitive concepts of Observable $O$ are introduced. Then the states $\rho$ are defined as a derived concept. They are the functional over the observable space. Then the "bra" and "kets" are simple vectors while the observables and states are matrices. Then somehow the usual treatment of quantum chaos in closed systems with the "bra" and "ket" is not enough, since this formalism does not consider open systems.

Moreover, as we explained in the introduction of [2], there are many ways to define quantum chaos. Among these ways, following part one, we will say that "a quantum system is chaotic if its classical limit is chaotic", i.e. the Michael Berry's quantum chaos definition [14]. In this way we have defined quantum chaos in the two steps of the Quantum Ergodic Hierarchy: Ergodic and Mixing [2. These steps are defined by their correspondent classical limit: they have a Cèsaro and a Weak limit respectively, that correspond to the limit of the Ergodic and Mixing systems of the classical ergodic hierarchy [1]. We will follow the same strategy with the other two levels.

Then, we have explained this limit in great detail. Precisely we have considered the WeylWigner-Moyal transformation [3], the transformation symb that changes quantum operators and states into the corresponding classical symbols which become operators and states of classical analytic Mechanics when $\hbar \rightarrow 0$.

$$
\text { symb } \widehat{O}=O(q, p), \quad \text { symb } \widehat{\rho}=\rho(q, p), \text { if } \hbar \rightarrow 0
$$

Then it can be demonstrated that

$$
\operatorname{Tr}(\widehat{\rho} \widehat{O})=\int_{\Gamma} \rho(q, p) O(q, p) d q d p
$$

where $\Gamma$ is the phase space, and the last equation is valid even if $\hbar \neq 0$.

Thus, we define a classical mixing system as the one that satisfies the weak limit

$$
\lim _{t \rightarrow \infty} \int_{\Gamma} \rho(q, p, t) O(q, p) d q d p=\int_{\Gamma} \rho_{*}(q, p) O(q, p) d q d p
$$

where $\rho_{*}(q, p)$ is the weak equilibrium state of $\rho(q, p, t)$ and $O(q, p)$ belongs to the space of observables. Then from (1) the corresponding quantum chaos will satisfy the weak limit

$$
\lim _{t \rightarrow \infty} \operatorname{Tr}(\widehat{\rho}(t) \widehat{O})=\operatorname{Tr}\left(\widehat{\rho}_{*} \widehat{O}\right)
$$


namely the definition of quantum mixing chaos that we have given in paper 2 section 6.3 , definition B.

This way to define the quantum version of the Ergodic Hierarchy, based in the classical one, is the one that we will also use in this paper (see Table II page 28).

As a consequence, following the ideas of 9 and the preceding paper 2, we will study the problem of quantum chaos hierarchy directly from the quantum description of the chaotic classical limit.

So as in paper [2], that we consider as the first part of this paper, we have defined the quantum chaos in the two first levels of the ergodic hierarchy (EH): ergodic and mixing. In this paper we will complete the work adding two more levels: Kolmogorov and Bernoulli. Then this paper can be considered as a second part of paper [2]. Nevertheless in this paper following the ideas of paper [1] we will first repeat the two initial levels using these new concepts and then adding the two final levels. Also, for the sake of conciseness, we will not repeat the following sections of paper [2] section 2 (Mathematical background), section 3 (Decoherence in non integral systems), section 4 (The classical statistical limit), and section 5 (The classical limit). These sections can be read in [2]. The just quoted section 5 can also be complemented with paper [15].

The paper is thus organized. Section 2: We present the formalism, definitions and the Ergodic Hierarchy (EH) which we will use. Section 3 and 4: we briefly review the ergodic and mixing systems already considered in ref. 2. Section 5 and 6: We explain the Kolmogorov and Bernoulli cases in detail. Section 7: we give a physical relevance analyzing two emblematic examples of the literature in terms of the Quantum Ergodic Hierarchy: the Casati-Prosen model [4, 5] and the kicked rotator [6, 7, 8]. Section 8: We consider the relevance of the subject and draw our conclusions.

\section{Formalism}

For a Hilbert space $\mathcal{H}$ of dimension $N>2$ the set of pure states forms a $(2 N-2)$-dimensional manifold, of measure zero, in the $\left(N^{2}-2\right)$-dimensional boundary $\partial \mathcal{C}_{N}$ of the set $\mathcal{C}_{N}$ of density matrices. The set of mixed quantum states $\mathcal{C}_{N}$ consists of Hermitian, positive matrices of size $N$, normalized by the trace condition, that is

$$
\mathcal{C}_{N}=\left\{\rho: \rho=\rho^{\dagger} ; \rho \geq 0 ; \operatorname{tr}(\rho)=1 ; \operatorname{dim}(\rho)=N\right\} .
$$

It can be shown for finite dimensional bipartite states that there exists always a non-zero measure $\mu_{s}$ in the neighborhood of separable states containing maximum uncertainty ones $\mu_{s}$ tends to zero as the dimension tends to infinity. Finally, for an infinitely dimensional Hilbert space almost all states are entangled [16, 17.

In this section we would like to establish the relation of three different levels:

- The notion of sets correlations, the main tool of paper [1].

- The algebra of the observables and states (symbolized by density or distribution functions) in the phase space of the Hamiltonian Mechanics (see [18] and [19]).

- The same algebra at quantum mechanics level obtained through the Weyl-Wigner-Moyal transformation form the Hamiltonian Mechanics (see [3] and [20] for details). 


\subsection{Definitions}

- Let $\left[X, \Sigma, \mu, T_{t}\right]$ be a generic dynamically system, where $X$ is a set, $\Sigma$ is a $\sigma$-algebra, $\mu$ is a measure, and $T_{t}$ is a time transformation. In Hamiltonian Mechanics, the physical case, $X$ is the phase space with coordinates $\phi=(q, p)$, (or a projection $\Pi X$ of phase space whose coordinates will also symbolize $\phi), \Sigma$ is the $\sigma$-algebra of measurable sets of $X, \mu$ is the Liouville measure $d \phi=d q d p$, (usually normalized as $\mu(X)=1$ ), and $T_{t}$ is given by the Hamiltonian dynamics.

- The $\Sigma$ algebra has the following properties:

(1) $X \in \Sigma$,

(2) $A \backslash B \in \Sigma$ for all $A, B \in \Sigma$, and

(3) $\bigcup_{i=1}^{n} B_{i} \in \Sigma$ if $B_{i} \in \Sigma$ for $1 \leq i \leq n \leq \infty$. As a consequence $\Sigma$ also contains $\varnothing$ and $\bigcap_{i=1}^{n} B_{i}$ if $B_{i} \in \Sigma$, for $1 \leq i \leq n \leq \infty$

- The probability measure $\mu$ on $\Sigma$ is such that

(1) $\mu: \Sigma \rightarrow 1$ with $\mu(X)=1$, and

(2) If $\left\{B_{i}\right\}_{i=1}^{n} \subset \Sigma$ and $B_{j} \cap B_{k}=\varnothing$ for $1 \leq j \leq k \leq n \leq$ then $\mu\left(\bigcup_{i=1}^{n} B_{i}\right)=\sum_{i=1}^{n} \mu\left(B_{i}\right)$.

- The automorphism $T$ is an automorphism that maps the probability space $[X, B, \mu]$ onto itself and it is measure preserving iff $B \in X$ i.e.:

(1) $T^{-1} B \in \Sigma$,

(2) $\mu\left(T^{-1} B\right)=\mu(B)$, where $T^{-1} B=[x \in X: T x \in B]$

- A dynamic law or time evolution $\tau=\left\{T_{t}\right\}_{t \in I}$ is a group of measure preserving authomorphisms $T_{t} X \rightarrow X$ of the probability space $[X, B, \mu]$ onto itself and where $I$ is either $\mathbb{R}$ or $\mathbb{Z}$.

- The set $\alpha=\left\{\alpha_{i}: i=1, \ldots, N\right)$ is a partition of $X$ iff

(1) $\alpha_{i} \cap \alpha_{j}=\varnothing$ for all $i \neq j$,

(2) $\mu\left(X \backslash \bigcup_{i=1}^{n}\right)=0$.

(3) Given two partitions $\alpha=\left\{\alpha_{i}: i=1, \ldots, N\right\}, \beta=\left\{\beta_{j}: j=1, \ldots, M\right\}$ we will call their $\operatorname{sum} \alpha \vee \beta=\left\{\alpha_{i} \cap \beta_{j}: i=1, \ldots, N ; j=1, \ldots, M\right\}$

- A $\sigma$-sub algebra $\Sigma_{0} \subset \Sigma$ must also satisfy the conditions

(1) $\Sigma_{0} \subseteq T \Sigma_{0}$

(2) $\vee_{n=-\infty}^{\infty} T^{n} \Sigma_{0}=\Sigma$,

(3) $\wedge_{n=-\infty}^{\infty} T^{n} \Sigma_{0}=N$ namely the $\sigma$-algebra containing the set of measure one and zerd!.

- Let $A$ and $B$ be measurable sets of the space $X$, and let $\mu$ be the measure just defined. Then the correlation between $A$ and $B$ is defined as

$$
C(B, A)=\mu(A \cap B)-\mu(A) \mu(B)
$$

\footnotetext{
${ }^{1}$ Of course, the last condition cannot be fulfilled in the quantum case because the phase space will have an intrinsic graininess originated in the uncertainty principle. So we must consider that this condition would only be approximately satisfied.
} 
Let us explain the meaning of this notion. In a generic system and under generic circumstances we have $C(B, A) \neq 0$. But if $C(B, A)=0$ some kind of "homogeneity" has appeared in the system since both factors $\mu(A)$ and $\mu(B)$ play the same role in the product $\mu(A \cap B)$, precisely:

$$
\mu(A \cap B)=\mu(A) \mu(B)
$$

This "homogenization" in the behavior of $\mu(A \cap B)$ corresponds to the vanishing of correlations. Then, if the time evolution $T_{t}$ conserves the measures or $\mu\left(T_{t} A\right)=\mu(A)$, (as in the phase space) we have

$$
\mu\left(T_{t} A \cap B\right)=\mu(A) \mu(B)+C\left(B, T_{t} A\right)
$$

where $\mu(A) \mu(B)$ would be the "homogenous" constant part of $\mu\left(T_{t} A \cap B\right)$ and $C\left(B, T_{t} A\right)$ the "non-homogenous" variable part. Then, if e. g., when $t \rightarrow \infty$ we have $C(B, A) \rightarrow 0$ some homogenization has taken place in the system 2 .

\subsection{The Ergodic Hierarchy (EH)}

Using the notion of correlation (see equations (3) and (5) ) we can define the main four steps of the EH as:

- Ergodic systems if

$$
\lim _{T \rightarrow \infty} \frac{1}{T} \int_{0}^{T} C\left(T_{t} B, A\right) d t=0
$$

We will call this limit a Cesàro limit.

- Mixing systems if

$$
\lim _{t \rightarrow \infty} C\left(T_{t} B, A\right)=0
$$

We will call this limit a weak limit.

- Kolmogorov systems. Using the Cornfeld-Fomin-Sinai theorem (see 21 page 283) the traditional definition for these system can be translated to the correlations' language as follows:

$A$ system is Kolmogorov (K-system) if for any integer $r$ and any set $A_{0}, A_{1}, A_{2}, . . A_{r} \in X$ and for any $\varepsilon>0$ there exists an $n_{0}>0$ such for all $B \in \sigma_{n, r}\left(A_{1}, A_{2}, . . A_{r}\right)$, we have

$$
\left|C\left(B, A_{0}\right)\right|<\varepsilon
$$

where $\sigma_{n, r}\left(A_{1}, A_{2}, . . A_{r}\right)$ is a sub $\sigma$-algebra (defined in section 2.1.vii)

For example this $\sigma$-algebra contains, among others, the following sets.

1. All the $T_{k} A_{i}$, for all $k \geq n$, and all $i=1, \ldots r$.

\footnotetext{
${ }^{2}$ Correlations are also related with the notion of unpredictability [1], but we do not consider this subject in this paper.
} 
2. All the finite and infinite sequences $T_{n} A_{m_{1}} \cup T_{n} A_{m_{2}} \cup T_{n} A_{m_{3}} \ldots$, and $T_{n} A_{m_{1}} \cup T_{n+1} A_{m_{2}} \cup$ $T_{n+2} A_{m_{3}} \ldots$ where $m_{i} \in(i=1, \ldots r)$

3. All the finite and infinite sequences $T_{n} A_{m_{1}} \cap T_{n} A_{m_{2}} \cap T_{n} A_{m_{3}} \ldots$ and $T_{n} A_{m_{1}} \cap T_{n+1} A_{m_{2}} \cap$ $T_{n+2} A_{m_{3}} \ldots$ where $m_{i} \in(i=1, \ldots r)$

- Bernoulli system If for any time $t$

$$
C\left(T_{t} B, A\right)=0
$$

so from eq. (5) if $\mu\left(T_{t} A\right)=\mu(A)$ we have

$$
\mu\left(T_{t} B \cap A\right)=\mu(A) \mu(B)
$$

i.e. in probability language: the probability to obtain the event $B$, at any time, conditioned by $A$ is always the same and we have the homogeneity defined in eq. (4).

Then the levels of the EH are defined by the way the correlations vanishes when $t \rightarrow \infty$ (being the Bernoulli level defined by a trivial zero identity).

\subsection{Correlations at the different levels}

Now we can also define the notion of correlation at their different levels of subsection 2.2.

I) Measurable set level

$$
C(B, A)=\mu(A \cap B)-\mu(A) \mu(B)
$$

II) Distribution or density function level

$$
C(g, f)=\langle f, g\rangle-\langle f, 1\rangle\langle 1, g\rangle
$$

where $f$ (and $g$ ) is a function over the phase space $X$ such that the integral $\int_{X} f(\phi) d \phi$ exists, $\langle f, g\rangle=\int_{X} f(\phi) g(\phi) d \phi$ and where $\phi=(q, p)$, are the coordinates at a point of $X$, so $\phi \in X$ and $d \phi=\mu(d \phi)=d q d p$.

III) Quantum level

$$
C(\widehat{g} \mid, \widehat{f})=(\widehat{f} \mid \widehat{g})-(\widehat{f} \mid \widehat{I})(\widehat{I} \mid \widehat{g})
$$

where $\widehat{f}, \widehat{g} \in \mathcal{A}$ the algebra of observables. Then if $f=\operatorname{symb}(\widehat{f} \mid$ and $g=\operatorname{symb}(\widehat{g} \mid$ are the Weyl-Wigner-Moyal transforms of $(\widehat{f} \mid$ and $\mid \widehat{g})$ we know that $(\widehat{f} \mid \widehat{g})=\langle f, g\rangle \mid 3$. Then using the usual quantum symbols for observables and states we have

$$
C(\widehat{O}, \widehat{\rho})=(\widehat{\rho} \mid \widehat{O})-(\widehat{\rho} \mid \widehat{I})(\widehat{I} \mid \widehat{O})
$$

where $\widehat{\rho}=\widehat{f}$ are the states and $\widehat{O}=\widehat{g}$ are the observables 4

From these equations we can see that we can translate the EH up to a Quantum Ergodic Hierarchy (QEH), we have done for the two first steps, for The Ergodic Hierarchy, in paper [2].

\footnotetext{
${ }^{3}$ In the process, from $I$ ) to $\left.I I I\right)$, we may say that the ignorance probabilities become intrinsic probabilities, but numerically they are equal.

${ }^{4}$ The normalization of $\widehat{\rho}(t)$ is simply $(\widehat{\rho} \mid \widehat{I})=1$ or $\operatorname{Tr} \widehat{\rho}=1$ so $C(\widehat{O}, \widehat{\rho})=(\widehat{\rho} \mid \widehat{O})-(\widehat{I} \mid \widehat{O})=(\widehat{\rho} \mid \widehat{O})-\operatorname{Tr} \widehat{O}$
} 
Let us now schematically show the relations among eqs., (11) to (14). Let us define the characteristic function $1_{A}(\phi)$ as

$$
1_{A}(\phi)=1 \text { if } \phi \in A, \quad 1_{A}(\phi)=0 \text { if } \phi \notin A
$$

Then as $1_{A}^{2}(\phi)=1_{A}(\phi)$, and $1_{A}(\phi)$ can also be considered as a projector $\Pi_{A}(\phi)=1_{A}(\phi)$. Using these projectors we can write the definition (11) as

$$
C(B, A)=\int_{X} 1_{A}(\phi) 1_{B}(\phi) d \phi-\int_{X} 1_{A}(\phi) d \phi \int_{X} 1_{B}(\phi) d \phi
$$

since it is evident that the terms of the r.h.s. of both equations are the same.

Let us now define a partition $\left\{A_{i}\right\}$ of $X$ that satisfies

$$
X=\bigcup_{i} A_{i}, \quad A_{i} \cap A_{i}=\emptyset \text { if } i \neq j
$$

or such that

$$
1_{A_{i}} 1_{A_{j}}=\delta_{i j} 1_{A_{i}}
$$

Let us also introduce two arbitrary sets of number $a_{i}, b_{j} \in \mathbb{R}$, then from eq. (15)

$$
\begin{gathered}
\sum_{i j} a_{i}, b_{j} C\left(A_{j}, A_{i}\right)= \\
=\sum_{i j} \int_{X} a_{i}, b_{j} 1_{A_{i}}(\phi) 1_{A_{j}}(\phi) d \phi-\sum_{i} \int_{X} a_{i} 1_{A_{i}}(\phi) d \phi \sum_{j} \int_{X} b_{j} 1_{A_{j}}(\phi) d \phi
\end{gathered}
$$

Then if we define two functions

$$
f(\phi)=\sum_{i} a_{i} 1_{A_{i}}(\phi), \quad g\left((\phi)=\sum_{j} b_{j} 1_{A_{j}}(\phi)\right.
$$

it is clear that since we can make the domains $A_{i}$ of the partition as small as we want we can approximate $f(\phi)$ and $g(\phi)$, then we can define

$$
C(g, f)=\sum_{i j} a_{i}, b_{j} C\left(A_{j}, A_{i}\right)=\int_{X} f(\phi) g(\phi) d \phi-\int_{X} f(\phi) d \phi \int_{X} g(\phi) d \phi
$$

or defining $\langle f(\phi), g(\phi)\rangle=\int_{X} f(\phi) g(\phi) d \phi$.

$$
C(g, f)=\sum_{i j} a_{i}, b_{j} C\left(A_{j}, A_{i}\right)=\langle f(\phi), g(\phi)\rangle-\langle f(\phi), 1\rangle\langle 1, g(\phi)\rangle
$$

i.e. the definition of correlations in the distribution function language (cf. eq. (12)) is demonstrated. This definition is equivalent to (11) if $a_{i}=\delta_{i 0}, b_{j}=\delta_{j 1}, A_{0}=B, A_{1}=A$.

Given $(\widehat{\rho} \mid \widehat{I})=\langle\rho, 1\rangle=\langle\rho\rangle$ and symb $\widehat{I}=1$, using to (17) the Weyl-Wigner-Moyal transform and interpreting $\widehat{f}$ as the state and $\widehat{g}$ as the operator, if symb $\widehat{O}=O(\phi)$ and symb $\widehat{\rho}=\rho(\phi)$, we have that 


$$
C(\widehat{O}, \widehat{\rho})=(\widehat{\rho} \mid \widehat{O})-(\widehat{\rho} \mid \widehat{I})(\widehat{I} \mid \widehat{O})
$$

i.e. the definition of correlations but now at the quantum language (cf. eq. (14)) which is equivalent to (17) from the properties of Weyl-Wigner-Moyal transform. So when $\hbar \rightarrow 0$ we have $(18) \Leftrightarrow(17) \Leftrightarrow(11)$.

So we can see that the three levels: measurable set level, distribution function level, and quantum level are all equivalent and interchangeable.

\subsection{More general equations and the Ergodic Hierarchy (EH)}

- We will call Frobenius-Perron operator $P_{t}$ to the evolution operator of distributions or density functions. In quantum language the Frobenius-Perron operator $P_{t}$ would be the evolution operator for states, while the Koopman operator $U_{t}$ would be the time evolution operator for observables. In fact we have that

$$
\left\langle P_{t} f, g\right\rangle=\left\langle f, U_{t} g\right\rangle
$$

see [18] eq.(3.3.4).

Then $P_{t}$, the Frobenius-Perron operator, conserves the measure. Then we have $\int_{X} P_{t} 1_{A_{i}} d \phi=$ $\int_{X} 1_{A_{i}} d \phi$ and $\sum_{i} a_{i} \int_{X} P_{t} 1_{A_{i}} d \phi=\sum_{i} a_{i} \int_{X} 1_{A_{i}}$ thus

$$
\int_{X} P_{t} f d \phi=\int_{X} f d \phi \text { or }\left\langle P_{t} f\right\rangle=\langle f\rangle
$$

or at the quantum level, since $\langle f\rangle=\langle f . I\rangle=(\widehat{f} \mid \widehat{I})=\operatorname{Tr} \widehat{f}$, we have

$$
\operatorname{Tr}(\widehat{\rho}(t))=\operatorname{Tr}(\widehat{\rho}(0))
$$

namely the trace is also conserved.

- In general there exists several $f_{*}$, the equilibrium distributions such, that $P_{t} f_{*}=f_{*}$. But if the system is ergodic there is only one of them, therefore we will only consider this case.

- At the two first levels of the EH we will have a limit (Cesàro, Mixing) $P_{t} f \rightarrow f_{*}$ when $t \rightarrow \infty$ and from this limit we will have $\left\langle f_{*}\right\rangle=\langle f(t)\rangle$ or $\operatorname{Tr} \widehat{\rho}_{*}=\operatorname{Tr} \rho(t)$, since the norm is also conserved at the limit.

Then we can define a new measure $\mu_{*}(A)$ such that

$$
\mu_{*}(A)=\int_{A} f_{*}(\phi) d \phi
$$

and define a new correlation

$$
C_{*}(B, A)=\mu_{*}(A \cap B)-\mu_{*}(A) \mu_{*}(B)
$$

Now we can define the new levels: Ergodic and Mixing making $\mu \rightarrow \mu_{*}$ in eqs. (6) to (9). So we have the Ergodic Hierarchy (EH). Then, e. g., in the mixing case (see [22] pag. 58) we have

$$
\lim _{t \rightarrow \infty} \mu_{*}\left(T_{t} A \cap B\right)=\mu_{*}(A) \mu_{*}(B)
$$


then

$$
\lim _{t \rightarrow \infty} \mu_{*}\left(T_{t} A \cap X\right)=\mu_{*}(A) \mu_{*}(X)
$$

and if we normalize $\mu_{*}(X)=1$.

$$
\lim _{t \rightarrow \infty} \mu_{*}\left(T_{t} A\right)=\mu_{*}(A)
$$

i.e. the conservation of the normalization is also valid at the limit $t \rightarrow \infty$.

- Let us quote the Theorem 5.1 of [22]:

"Let $T_{t}$ be an ergodic transformation, with stationary density $f_{*}(\phi)$ of the associated Frobenius-Perron operator, operating in a phase space of finite $\mu_{*}$ measure. Then $T_{t}$ is mixing iff $\left\{P_{t} f\right\}$ is weakly convergent to $f_{*}(\phi)$ for all densities $f$, i. e.

$$
\lim _{t \rightarrow \infty}\left\langle P_{t} f, g\right\rangle=\left\langle f_{*}, g\right\rangle
$$

for every bounded measurable function $g$ ".

The demonstration is:

$$
\begin{aligned}
& \lim _{t \rightarrow \infty} \mu_{*}\left(T_{t} A \cap B\right)=\lim _{t \rightarrow \infty} \int_{T_{t} A \cap B} f_{*}(\phi) d \phi=\lim _{t \rightarrow \infty} \int_{X} 1_{T_{t} A \cap B} f_{*}(\phi) d \phi= \\
& =\lim _{t \rightarrow \infty} \int_{X} 1_{T_{t} A} 1_{B} f_{*}(\phi) d \phi=\lim _{t \rightarrow \infty}\left\langle P_{t} 1_{A} f_{*}(\phi), 1_{B}\right\rangle
\end{aligned}
$$

and also

$$
\mu_{*}(A) \mu_{*}(B)=\int_{X} 1_{A} f_{*}(\phi) d \phi \int_{X} 1_{B} f_{*}(\phi) d \phi=\left\langle 1_{A} f_{*}(\phi), 1\right\rangle\left\langle f_{*}(\phi), 1_{B}\right\rangle
$$

so from eq. (22) we have

$$
\lim _{t \rightarrow \infty}\left\langle P_{t} 1_{A} f_{*}(\phi), 1_{B}\right\rangle=\left\langle 1_{A} f_{*}(\phi), 1\right\rangle\left\langle f_{*}(\phi), 1_{B}\right\rangle
$$

or

$$
\lim _{t \rightarrow \infty}\left\langle P_{t} 1_{A_{i}} f_{*}(\phi), 1_{B_{j}}\right\rangle=\left\langle 1_{A_{i}} f_{*}(\phi), 1\right\rangle\left\langle f_{*}(\phi), 1_{B_{j}}\right\rangle
$$

so considering two sets of generic numbers $\left(a_{i}\right)$ and $\left(b_{j}\right)$ and define the generic functions

$$
f=\sum_{i} a_{i} 1_{A_{i}} f_{*}(\phi), \quad g=\sum_{j} b_{j} 1_{B_{j}},
$$

we obtain

$$
\lim _{t \rightarrow \infty}\langle f, g\rangle=\langle f, 1\rangle\left\langle f_{*}(\phi), g\right\rangle
$$

and if $f$ is normalized as $\langle f, 1\rangle=1$ the thesis follows. q.e.d.

Or in other words,

$$
W-\lim _{t \rightarrow \infty} P_{t} f=f_{*}
$$


Finally the corresponding definition of quantum mixing is

$$
\lim _{t \rightarrow \infty}(\widehat{\rho}(t) \mid \widehat{O})=\left(\widehat{\rho}_{*} \mid \widehat{O}\right)
$$

namely $\widehat{\rho}(t)$ weakly converges to $\widehat{\rho}_{*}$ (see [2]).

For the ergodic case we must simply make the substitution $\lim _{t \rightarrow \infty} \rightarrow \lim _{t \rightarrow \infty} \frac{1}{T} \int_{0}^{T}$ or $\lim _{n \rightarrow \infty} \frac{1}{n} \sum_{0}^{n-1}$ in the discrete case. The Kolmogorov and Bernoulli cases will be considered in sections 5 and 6 . In Table I we display the synthetic structure of the three levels.

TABLE I: SET LEVEL, DISTRIBUTION FUNCTION LEVEL, QUANTUM LEVEL

\section{SETS FUNCTIONS Q QUANTUM OPERATORS}

$\begin{array}{lccc}\begin{array}{l}\text { EVOLUTION } \\ \text { (projectors) }\end{array} & \begin{array}{c}A \rightarrow T A \\ \text { Liouville ev. }\end{array} & \begin{array}{c}1_{A} \rightarrow P_{t} 1_{A}=1_{T_{A}} \\ \text { Frob.-Perron ev. }\end{array} & \begin{array}{c}\text { symb } \\ \text { Heisenberg ev. }\end{array} 1_{T_{A}}=\widehat{P}_{A}(t) \\ \begin{array}{l}\text { EQUILIBRIUM } \\ \text { (states) }\end{array} & & \begin{array}{c}U_{t} f_{*}=f_{*} \\ \text { Koopman ev. }\end{array} & \begin{array}{c}\widehat{U}_{t} \widehat{\rho}_{*} \widehat{U}_{t}^{\dagger}=\widehat{\rho}_{*} \\ \text { Schroedinger ev. }\end{array} \\ \text { OPERATIONS } & A \cap B & 1_{A} 1_{B} & \widehat{P}_{A} \widehat{P}_{B}, \hbar \sim 0 \\ & A \cup B & 1_{A}+1_{B}-1_{A} 1_{B} & \widehat{P}_{A}+\widehat{P}_{B}-\widehat{P}_{A} \widehat{P}_{B}, \hbar \sim 0\end{array}$

\section{Ergodic Systems}

According to paper [1] eq. (E) the system is uniformly ergodic if

$$
\lim _{n \rightarrow \infty} \frac{1}{n} \sum_{k=0}^{n-1} \mu\left(T_{k} B \cap A\right)=\mu(A) \mu(B)
$$

or

$$
\lim _{n \rightarrow \infty} \frac{1}{n} \sum_{k=0}^{n-1} C\left(T_{k} B, A\right)=0
$$

But if we introduce the measure $\mu_{*}(A)$, as we have defined the new Ergodic level making $\mu \rightarrow \mu_{*}$, we have that the system is ergodic if

$$
\lim _{n \rightarrow \infty} \frac{1}{n} \sum_{k=0}^{n-1} \mu_{*}\left(T_{k} A \cap B\right)=\mu_{*}(A) \mu_{*}(B)
$$

or for the distribution of density function case (see also the corresponding theorem 4.7 in [22]) or in the continuous case

$$
\lim _{T \rightarrow \infty} \frac{1}{T} \int_{0}^{T}\left\langle P_{t} f, g\right\rangle d t=\left\langle f_{*}, g\right\rangle
$$


or finally in the quantum case, it is quantum ergodic if

$$
\lim _{T \rightarrow \infty} \frac{1}{T} \int_{0}^{T}(\widehat{\rho}(t) \mid \widehat{O}) d t=\left(\widehat{\rho}_{*} \mid \widehat{O}\right)
$$

as explained in all details in the first part of this paper i.e. 2]. We also include the discrete version of the quantum ergodic because in many situations the evolution of chaotic systems is given in terms of a discrete evolution operator, for example when the Hamiltonian has a discrete symmetry (see for example the Floquet systems in [6]).

$$
\lim _{N \rightarrow \infty} \frac{1}{N} \sum_{k=0}^{N-1}(\widehat{\rho}(k) \mid \widehat{O}) d t=\left(\widehat{\rho}_{*} \mid \widehat{O}\right)
$$

In section 7 we apply this discrete version to the kicked rotator.

\section{Mixing Systems}

According to paper [1] eq. (M) the system is mixing if

$$
\lim _{n \rightarrow \infty} \mu\left(T_{n} B \cap A\right)=\mu(A) \mu(B)
$$

or

$$
\lim _{n \rightarrow \infty} C\left(T_{n} B, A\right)=0
$$

Moreover from [22] page 58 the system is mixing if

$$
\lim _{n \rightarrow \infty} \mu_{*}\left(T_{n} A \cap B\right)=\mu_{*}(A) \mu_{*}(B)
$$

or for the distribution of density function case (also see the corresponding theorem 5.1 in [22])

$$
\left\langle P_{t} f, g\right\rangle=\left\langle f_{*}, g\right\rangle
$$

or in the quantum case, it is quantum mixing if

$$
\lim _{t \rightarrow \infty}(\widehat{\rho}(t) \mid \widehat{O})=\left(\widehat{\rho}_{*} \mid \widehat{O}\right)
$$

as explained in all details in the first part i.e. 2.

In some cases it might be easier to demonstrate that a system is mixing using a discrete evolution. The corresponding discrete version of (34) is

$$
\lim _{N \rightarrow \infty}(\widehat{\rho}(N) \mid \widehat{O})=\left(\widehat{\rho}_{*} \mid \widehat{O}\right)
$$

\section{Kolmogorov Systems}

The two previous sections are essentially contained in [2] and they were introduced here for the sake of completeness. This section is the most technical part of the paper. We remark that things are not so simple at the Kolmogorov level essentially because the theorem in section 2.4 cannot be reproduced. We begin by recalling the definition of Kolmogorov systems at the measurable set level. 


\subsection{Kolmogorov systems in the EH}

We return to the definition of Kolmogorov systems of subsection 2.2:

$A$ system is Kolmogorov (K-system) if for any integer $r$ and any set $A_{0}, A_{1}, A_{2}, . . A_{r} \in X$ and for any $\varepsilon>0$ there exists an $n_{0}>0$ such for all $B \in \sigma_{n, r}\left(A_{1}, A_{2}, . . A_{r}\right)$ and any $n>n_{0}$ we have

$$
\left|C\left(B, A_{0}\right)\right|<\varepsilon
$$

Then,

$$
\lim _{n \rightarrow \infty} C\left(B, A_{0}\right)=\lim _{n \rightarrow \infty}\left\{\mu\left(B \cap A_{0}\right)-\mu(B) \mu\left(A_{0}\right)\right\}=0 \quad \forall B \in \sigma_{n, r}\left(A_{1}, A_{2}, \ldots, A_{r}\right)
$$

where $\sigma_{n, r}\left(A_{1}, A_{2}, \ldots, A_{r}\right)$ is the $\sigma$-algebra generated by $\left\{T^{k} A_{i}: k \geq n ; i=1, \ldots, r\right\}$, and therefore $\sigma_{n, r}\left(A_{1}, A_{2}, \ldots, A_{r}\right)=\sigma\left(\left\{T^{k} A_{i}: k \geq n ; i=1, \ldots, r\right\}\right)$

Recall that if $f_{*}$ is an stationary density, namely $P_{t} f_{*}=f_{*}$ then the measure $\mu_{*}$ given by

$$
\mu_{*}(A)=\int_{A} f_{*}(\phi) d \phi \quad \forall A \in X
$$

is an invariant measure (i.e. $\mu_{*}\left(S^{-1}(A)\right)=\mu_{*}(A)$ for all transformation $S: X \rightarrow X$ and for all $A \in X)$ (see Theorem 4.1.1. of [18]).

As we consider the previous sections, we make $\mu=\mu_{*}$ and therefore the Kolmogorov condition (37) becomes

$$
\lim _{n \rightarrow \infty}\left\{\mu_{*}\left(B \cap A_{0}\right)-\mu_{*}(B) \mu_{*}\left(A_{0}\right)\right\}=0 \quad \forall B \in \sigma_{n, r}\left(A_{1}, A_{2}, \ldots, A_{r}\right)
$$

Now a question arises, What are the sets containing the $\sigma$-algebra $\sigma\left(\left\{T^{k} A_{i}: k \geq n ; i=\right.\right.$ $1, \ldots, r\})$ ? There are two types of these sets:

(I) $B=\bigcup_{i} T_{n+n_{i}} A_{s_{i}} \backslash T_{n+l_{i}} A_{p_{i}} \quad$ (finite or countable unions of $T_{i} A_{i} \backslash T_{j} A_{j}$ )

(II) $B=\bigcap_{i} T_{n+n_{i}} A_{s_{i}}$ (finite or countable intersections of $T_{i} A_{i}$ )

where $n_{i}, l_{i} \in \mathbb{N}_{0}$ and $s_{i}, p_{i} \in\{1, \ldots, r\}$.

It is clear that (finite or countable) unions of $T_{i} A_{i}$ are included because it is sufficient to make in $(I) A_{p_{i}}=\emptyset$ for all $p_{i}$ and results $B=\bigcup T_{n+n_{i}} A_{s_{i}}$.

Therefore, if we can translate the condition (39) into quantum language for the sets of type $(I)$ and $(I I)$ we will have the Kolmogorov Quantum Hierarchy in the UEH. We begin with the sets of type $(I)$ :

We have that for these type of sets the condition (39) becomes

$$
\lim _{n \rightarrow \infty}\left\{\mu_{*}\left(\bigcup_{i} T_{n+n_{i}} A_{s_{i}} \backslash T_{n+l_{i}} A_{p_{i}} \cap A_{0}\right)-\mu_{*}\left(\bigcup_{i} T_{n+n_{i}} A_{s_{i}} \backslash T_{n+l_{i}} A_{p_{i}}\right) \mu_{*}\left(A_{0}\right)\right\}
$$

which is equal to

$$
\lim _{n \rightarrow \infty}\left\{\mu_{*}\left(\bigcup_{i} T_{n+n_{i}} A_{s_{i}} \cap\left(T_{n+l_{i}} A_{p_{i}}\right)^{c} \cap A_{0}\right)-\mu_{*}\left(\bigcup_{i} T_{n+n_{i}} A_{s_{i}} \cap\left(T_{n+l_{i}} A_{p_{i}}\right)^{c}\right) \mu_{*}\left(A_{0}\right)\right\}
$$


Now by the inclusion-exclusion principle (see for example [23]) if $P$ is a measure of probability and $Z_{1}, Z_{2}, Z_{3}, \ldots, Z_{n}$ are sets, then

$$
P\left(\bigcup_{i=1}^{n} Z_{i}\right)=\sum_{k=1}^{n} \sum_{I \subseteq\{1, \ldots, n\}, \sharp(I)=k}(-1)^{k+1} P\left(\bigcap_{i \in I} Z_{i}\right)
$$

where $P$ is the probability which extended for $n \rightarrow \infty$ it becomes

$$
P\left(\bigcup_{i=1}^{\infty} Z_{i}\right)=\sum_{k=1}^{\infty} \sum_{I \subseteq \mathbb{N}, \sharp(I)=k}(-1)^{k+1} P\left(\bigcap_{i \in I} Z_{i}\right)
$$

Since that $f_{*}$ is a density, more precisely $f_{*} \in D(X, \Sigma, \mu)=\left\{f \in L^{1}(X, \Sigma, \mu): f \geq 0 ;\|f\|=1\right\}$ (see Definition 3.1.3. of [18]), that is, $D(X, \Sigma, \mu)$ is the space of the distribution functions defined over all phase space. Then $\mu_{*}$ is a measure of probability and we can use (43) to express (41) as

$$
\begin{aligned}
& \lim _{n \rightarrow \infty} \sum_{k=1}^{\infty} \sum_{I \subseteq \mathbb{N}, \sharp(I)=k}(-1)^{k+1} \mu_{*}\left(\bigcap_{j \in I} T_{n+n_{j}} A_{s_{j}} \cap\left(T_{n+l_{j}} A_{p_{j}}\right)^{c} \cap A_{0}\right)- \\
& -\lim _{n \rightarrow \infty} \sum_{k=1}^{\infty} \sum_{I \subseteq \mathbb{N}, \sharp(I)=k}(-1)^{k+1} \mu_{*}\left(\bigcap_{j \in I} T_{n+n_{j}} A_{s_{j}} \cap\left(T_{n+l_{j}} A_{p_{j}}\right)^{c}\right) \mu_{*}\left(A_{0}\right)= \\
& =\lim _{n \rightarrow \infty} \sum_{k=1}^{\infty} \sum_{I \subseteq \mathbb{N}, \sharp(I)=k}(-1)^{k+1}\left\{\mu_{*}\left(\bigcap_{j \in I} T_{n+n_{j}} A_{s_{j}} \cap\left(T_{n+l_{j}} A_{p_{j}}\right)^{c} \cap A_{0}\right)-\right. \\
& \left.-\mu_{*}\left(\bigcap_{j \in I} T_{n+n_{j}} A_{s_{j}} \cap\left(T_{n+l_{j}} A_{p_{j}}\right)^{c}\right) \mu_{*}\left(A_{0}\right)\right\}= \\
& =\lim _{n \rightarrow \infty} \sum_{k=1}^{\infty} \sum_{I \subseteq \mathbb{N}, \mathbb{H}(I)=k}(-1)^{k+1} C_{*}\left(\bigcap_{j \in I} T_{n+n_{j}} A_{s_{j}} \cap\left(T_{n+l_{j}} A_{p_{j}}\right)^{c}, A_{0}\right)= \\
& =\sum_{k=1}^{\infty} \sum_{I \subseteq \mathbb{N}, \sharp(I)=k}(-1)^{k+1} \lim _{n \rightarrow \infty} C_{*}\left(\bigcap_{j \in I} T_{n+n_{j}} A_{s_{j}} \cap\left(T_{n+l_{j}} A_{p_{j}}\right)^{c}, A_{0}\right)=0
\end{aligned}
$$

where we have used $C_{*}(A, B)=\mu_{*}(A \cap B)-\mu_{*}(A) \mu_{*}(B)$. From the last equation (44) we see that the problem reduces to determining if the limit

$$
\lim _{n \rightarrow \infty} C_{*}\left(\bigcap_{j \in I} T_{n+n_{j}} A_{s_{j}} \cap\left(T_{n+l_{j}} A_{p_{j}}\right)^{c}, A_{0}\right)=0
$$

exists. So if we translate (45) to quantum language the resultant condition will be the fundamental property of the quantum Kolmogorov systems (because if we make $A_{p_{i}}=\emptyset$ for all $p_{i}$ then we obtain the condition of the sets of type $(I I))$. In a more general way we consider infinite numerable intersections 


$$
\begin{aligned}
& \lim _{n \rightarrow \infty} C_{*}\left(\bigcap_{j=1}^{\infty} T_{n+n_{j}} A_{s_{j}} \cap\left(T_{n+l_{j}} A_{p_{j}}\right)^{c}, A_{0}\right)=\lim _{n \rightarrow \infty}\left\{\mu_{*}\left(\bigcap_{j=1}^{\infty} T_{n+n_{j}} A_{s_{j}} \cap\left(T_{n+l_{j}} A_{p_{j}}\right)^{c} \cap A_{0}\right)-\right. \\
& \left.-\mu_{*}\left(\bigcap_{j=1}^{\infty} T_{n+n_{j}} A_{s_{j}} \cap\left(T_{n+l_{j}} A_{p_{j}}\right)^{c}\right) \mu_{*}\left(A_{0}\right)\right\}=0
\end{aligned}
$$

Now using the definition of $\mu_{*}$ (see equation (38)) the equation (46) is expressed as

$$
\begin{aligned}
& \lim _{n \rightarrow \infty}\left\{\int_{\bigcap_{j=1}^{\infty} T_{n+n_{j}} A_{s_{j}} \cap\left(T_{n+l_{j}} A_{p_{j}}\right)^{c} \cap A_{0}} f_{*}(\phi) d \phi-\right. \\
& \left.-\left(\int_{\bigcap_{j=1}^{\infty} T_{n+n_{j}} A_{s_{j}} \cap\left(T_{n+l_{j}} A_{p_{j}}\right)^{c}} f_{*}(\phi) d \phi\right)\left(\int_{A_{o}} f_{*}(\phi) d \phi\right)\right\}= \\
& =\lim _{n \rightarrow \infty}\left\{\int_{X} 1_{\bigcap_{j=1}^{\infty} T_{n+n_{j}} A_{s_{j}} \cap\left(T_{n+l_{j}} A_{p_{j}}\right)^{c} \cap A_{0}} f_{*}(\phi) d \phi-\right. \\
& \left.-\left(\int_{X} 1 \bigcap_{j=1}^{\infty} T_{n+n_{j}} A_{s_{j}} \cap\left(T_{n+l_{j}} A_{p_{j}}\right)^{c} f_{*}(\phi) d \phi\right)\left(\int_{X} f_{*}(\phi) 1_{A_{0}} d \phi\right)\right\}=0
\end{aligned}
$$

which is equal to

$$
\begin{aligned}
& \lim _{n \rightarrow \infty}\left\{\int_{X} \prod_{j=1}^{\infty} 1_{T_{n+n_{j}} A_{s_{j}}}\left(1-1_{T_{n+l_{j}} A_{p_{j}}}\right) f_{*}(\phi) 1_{A_{0}} d \phi-\right. \\
& \left.-\left(\int_{X} \prod_{j=1}^{\infty} 1_{T_{n+n_{j}} A_{s_{j}}}\left(1-1_{T_{n+l_{j}} A_{p_{j}}}\right) f_{*}(\phi) d \phi\right)\left(\int_{X} f_{*}(\phi) 1_{A_{0}} d \phi\right)\right\}=0
\end{aligned}
$$

Moreover the characteristic functions $1_{T_{n+n_{j}} A_{s_{j}}}, 1_{T_{n+l_{j}} A_{p_{j}}}$ are equal to $P_{n+n_{j}} 1_{A_{s_{j}}}, P_{n+l_{j}} 1_{A_{p_{j}}}$ respectively. Then (48) becomes

$$
\begin{aligned}
& \lim _{n \rightarrow \infty}\left\{\int_{X} \prod_{j=1}^{\infty} P_{n+n_{j}} 1_{A_{s_{j}}}\left(1-P_{n+l_{j}} 1_{A_{p_{j}}}\right) f_{*}(\phi) 1_{A_{0}} d \phi-\right. \\
& \left.-\left(\int_{X} \prod_{j=1}^{\infty} P_{n+n_{j}} 1_{A_{s_{j}}}\left(1-P_{n+l_{j}} 1_{A_{p_{j}}}\right) f_{*}(\phi) d \phi\right)\left(\int_{X} f_{*}(\phi) 1_{A_{0}} d \phi\right)\right\}=0
\end{aligned}
$$

Using that

$$
1-P_{n+l_{j}} 1_{A_{p_{j}}}=P_{n+l_{j}}\left(1-1_{A_{p_{j}}}\right)=P_{n+l_{j}} 1_{\left(A_{p_{j}}\right)^{c}}
$$

we see that the equation (49) can be expressed as 


$$
\begin{aligned}
& \lim _{n \rightarrow \infty}\left\{\int_{X} \prod_{j=1}^{\infty} P_{n+n_{j}} 1_{A_{s_{j}}} P_{n+l_{j}} 1_{\left(A_{p_{j}}\right)^{c}} f_{*}(\phi) 1_{A_{0}} d \phi-\right. \\
& \left.-\left(\int_{X} \prod_{j=1}^{\infty} P_{n+n_{j}} 1_{A_{s_{j}}} P_{n+l_{j}} 1_{\left(A_{p_{j}}\right)^{c}} f_{*}(\phi) d \phi\right)\left(\int_{X} f_{*}(\phi) 1_{A_{0}} d \phi\right)\right\}=0
\end{aligned}
$$

and therefore with the same trick used in subsection 2.4 we have

$$
\begin{aligned}
& \lim _{n \rightarrow \infty}\left\{\left\langle\prod_{j=1}^{\infty} P_{n+n_{j}} 1_{A_{s_{j}}} P_{n+l_{j}} 1_{\left(A_{p_{j}}\right)^{c}} f_{*}(\phi), 1_{A_{0}}\right\rangle-\right. \\
& \left.-\left\langle\prod_{j=1}^{\infty} P_{n+n_{j}} 1_{A_{s_{j}}} P_{n+l_{j}} 1_{\left(A_{p_{j}}\right)^{c}} f_{*}(\phi), 1\right\rangle\left\langle f_{*}(\phi), 1_{A_{0}}\right\rangle\right\}=0
\end{aligned}
$$

Then, we consider three sets of generic numbers $\left(a_{n}^{(j)}\right),\left(b_{m}^{(j)}\right)$ and $\left(c_{l}\right)$ and we define the generic functions

$$
\begin{aligned}
& f_{s_{j}}=\sum_{n} a_{n}^{(j)} 1_{A_{s_{j}}^{(n)}} \\
& f_{p_{j}}=\sum_{m} b_{m}^{(j)} 1_{\left(\left(A_{p_{j}}\right)^{c}\right)^{(m)}} f_{*}(\phi) \\
& g=\sum_{l} c_{l} 1_{A_{0}^{(l)}}
\end{aligned}
$$

Then we obtain that

$$
\begin{aligned}
& \lim _{n \rightarrow \infty}\left\{\left\langle\prod_{j=1}^{\infty} P_{n+n_{j}} f_{s_{j}} P_{n+l_{j}} f_{p_{j}}, g\right\rangle-\right. \\
& \left.-\left\langle\prod_{j=1}^{\infty} P_{n+n_{j}} f_{s_{j}} P_{n+l_{j}} f_{p_{j}}, 1\right\rangle\left\langle f_{*}(\phi), g\right\rangle\right\}=0
\end{aligned}
$$

If we realign the indices $n_{j}, l_{j}$ and define the functions $f_{s_{j}}, f_{p_{j}}$ such that $n_{j}=m_{j}, l_{j}=m_{j+1}$; $F_{j}=f_{s_{j}}, F_{j+1}=f_{p_{j}}$, that is, $f_{s_{j}}$ and $f_{p_{j}}$ are the $F_{j}$ terms of even and odd index. We have

$$
\lim _{n \rightarrow \infty}\left\{\left\langle\prod_{j=1}^{\infty} P_{n+m_{j}} F_{j}, g\right\rangle-\left\langle\prod_{j=1}^{\infty} P_{n+m_{j}} F_{j}, 1\right\rangle\left\langle f_{*}(\phi), g\right\rangle\right\}=0
$$

We can rewrite (55) as 


$$
\lim _{n \rightarrow \infty}\left\{\left\langle P_{n+m_{1}} F_{1}, g \prod_{j=2}^{\infty} P_{n+m_{j}} F_{j}\right\rangle-\left\langle P_{n+m_{1}} F_{1}, \prod_{j=2}^{\infty} P_{n+m_{j}} F_{j}\right\rangle\left\langle f_{*}(\phi), g\right\rangle\right\}=0
$$

Now according to paper [2] the star product tends to the product function when $\hbar \rightarrow 0$

$$
f(\phi) g(\phi) \longrightarrow(f * g)(\phi)=\operatorname{symb}(\widehat{f}) * \operatorname{symb}(\widehat{g})=\operatorname{symb}(\widehat{f} \widehat{g})
$$

and therefore when $\hbar \rightarrow 0$ for an infinite product of functions we have

$$
\begin{aligned}
& f_{i}(\phi)=\operatorname{symb}\left(\widehat{f}_{i}\right) \\
& \prod_{i=1}^{\infty} f_{i}(\phi)=\prod_{i=1}^{\infty} \operatorname{symb}\left(\widehat{f}_{i}\right)=\operatorname{symb}\left(\prod_{i=1}^{\infty} \widehat{f}_{i}\right)
\end{aligned}
$$

On the other hand from table 1 (subsection 2.4) we have

$$
P_{t} 1_{A}=1_{T_{t} A}=\operatorname{symb}\left(\widehat{P}_{A}(t)\right)
$$

and therefore, if we have a generic function $h$

$$
h=\sum_{k} h_{k} 1_{A_{k}}
$$

then (see eq. (22) of paper [2] and table 1 of subsection 2.4)

$$
\begin{aligned}
& \operatorname{symb}^{-1}\left(1_{A_{k}}\right)=\widehat{P}_{A_{k}} \\
& \widehat{h}=\operatorname{symb}^{-1}(h)=\sum_{k} h_{k} \operatorname{symb}^{-1}\left(1_{A_{k}}\right)=\sum_{k} h_{k} \widehat{P}_{A_{k}} \\
& \widehat{h}(t)=\sum_{k} h_{k} \widehat{P}_{A_{k}}(t) \\
& P_{t} h=\sum_{k} h_{k} P_{t} 1_{A_{k}}=\sum_{k} h_{k} P_{t} 1_{A_{k}}=\sum_{k} h_{k} P_{t} 1_{A_{k}}=\sum_{k} h_{k} \operatorname{symb}\left(\widehat{P}_{A_{k}}(t)\right)= \\
& =\operatorname{symb}\left(\sum_{k} h_{k} \widehat{P}_{A_{k}}(t)\right)=\operatorname{symb}(\widehat{h}(t))
\end{aligned}
$$

Then if we introduce the last equation of (58) and the equation (55) $P_{n+m_{j}} F_{j}$ (when $\hbar \rightarrow 0$ ) becomes

$$
\begin{aligned}
& P_{n+m_{j}} F_{j}=\operatorname{symb}\left(\widehat{F}_{j}\left(n+m_{j}\right)\right) \\
& \prod_{j=2}^{\infty} P_{n+m_{j}} F_{j}=\prod_{j=2}^{\infty} \operatorname{symb}\left(\widehat{F}_{j}\left(n+m_{j}\right)\right)=\operatorname{symb}\left(\prod_{j=2}^{\infty} \widehat{F}_{j}\left(n+m_{j}\right)\right)
\end{aligned}
$$

Now we call 


$$
\begin{aligned}
& f_{*}=\operatorname{symb}\left(\widehat{\rho}_{*}\right) \\
& g=\operatorname{symb}(\widehat{g})
\end{aligned}
$$

where $\widehat{\rho}_{*}$ is the weak limit of $\widehat{\rho}(t)$ (see [2]).

Therefore if we use (58), (62) and (63) in (56) when $\hbar \rightarrow 0$ we have

$$
\begin{aligned}
& \lim _{n \rightarrow \infty}\left\{\left\langle\operatorname{symb}\left(\widehat{F}_{1}\left(n+m_{1}\right)\right), \operatorname{symb}\left(\widehat{g} \prod_{j=2}^{\infty} \widehat{F}_{j}\left(n+m_{j}\right)\right)\right\rangle\right. \\
& \left.-\left\langle\operatorname{symb}\left(\widehat{F}_{1}\left(n+m_{1}\right)\right), \operatorname{symb}\left(\prod_{j=2}^{\infty} \widehat{F}_{j}\left(n+m_{j}\right)\right)\right\rangle\left\langle\operatorname{symb}\left(\rho_{*}\right), \operatorname{symb}(\widehat{g})\right\rangle\right\}=0
\end{aligned}
$$

Now this equation can be expressed in the quantum level as (replacing $\langle$,$\rangle by (\mid)$ )

$$
\begin{aligned}
& \lim _{n \rightarrow \infty}\left\{\left(\operatorname{symb}\left(\widehat{F}_{1}\left(n+m_{1}\right)\right) \mid \operatorname{symb}\left(\widehat{g} \prod_{j=2}^{\infty} \widehat{F}_{j}\left(n+m_{j}\right)\right)\right)\right. \\
& \left.-\left(\operatorname{symb}\left(\widehat{F}_{1}\left(n+m_{1}\right)\right) \mid \operatorname{symb}\left(\prod_{j=2}^{\infty} \widehat{F}_{j}\left(n+m_{j}\right)\right)\right)\left(\operatorname{symb}\left(\rho_{*}\right) \mid \operatorname{symb}(\widehat{g})\right)\right\}=0
\end{aligned}
$$

At this point we rename the operators $\widehat{F}_{1}\left(n+m_{1}\right)$, as $\widehat{F}_{j}\left(n+m_{j}\right)$ and $\widehat{g}$ as $\widehat{\rho}\left(n+m_{1}\right), \widehat{O}_{j}\left(n+m_{j}\right)$ and $\widehat{O}_{1}$ respectively. That is, if we emphasize the role of the states and the observables we have

$$
\begin{aligned}
& \lim _{n \rightarrow \infty}\left\{\left(\operatorname{symb}\left(\widehat{\rho}\left(n+m_{1}\right)\right) \mid \operatorname{symb}\left(\widehat{O}_{1} \prod_{j=2}^{\infty} \widehat{O}_{j}\left(n+m_{j}\right)\right)\right)\right. \\
& \left.-\left(\operatorname{symb}\left(\widehat{\rho}\left(n+m_{1}\right)\right) \mid \operatorname{symb}\left(\prod_{j=2}^{\infty} \widehat{O}_{j}\left(n+m_{j}\right)\right)\right)\left(\operatorname{symb}\left(\rho_{*}\right) \mid \operatorname{symb}\left(\widehat{O}_{1}\right)\right)\right\}=0
\end{aligned}
$$

Then, using the important property that the Wigner transformation yields the correct expectation value of any observable $\widehat{O}$ in the state $\widehat{\rho}$ (see equation (23) of paper [2]) we have

$$
\lim _{n \rightarrow \infty}\left\{\left(\widehat{\rho}\left(n+m_{1}\right) \mid \widehat{O}_{1} \prod_{j=2}^{\infty} \widehat{O}_{j}\left(n+m_{j}\right)\right)-\prod_{j=2}^{\infty}\left(\widehat{\rho}\left(n+m_{1}\right) \mid \widehat{O}_{j}\left(n+m_{j}\right)\right)\left(\widehat{\rho}_{*} \mid \widehat{O}_{1}\right)\right\}=0
$$

Finally, the definition of the quantum Kolmogorov level is

$$
\lim _{n \rightarrow \infty}\left\{\left(\widehat{\rho}\left(n+m_{1}\right) \mid \widehat{O}_{1} \prod_{j=2}^{\infty} \widehat{O}_{j}\left(n+m_{j}\right)\right)-\prod_{j=2}^{\infty}\left(\widehat{\rho}\left(n+m_{1}\right) \mid \widehat{O}_{j}\left(n+m_{j}\right)\right)\left(\widehat{\rho}_{*} \mid \widehat{O}_{1}\right)\right\}=0
$$

for all observables $\widehat{O}_{2}, \widehat{O}_{3}, \widehat{O}_{4}, \ldots$ and all $m_{1}, m_{2}, m_{3}, \ldots \in \mathbb{N}_{0}$ where $\widehat{\rho_{*}}$ is the weak limit of $\widehat{\rho}(t)$. 


\subsection{Particular Case: Mixing}

According to the definition of Kolmogorov level, we know that the mixing level includes Kolmogorov level, that is, the equation (37) implies the equation (31). Therefore it would be expected for a good definition of quantum Kolmogorov level given by equation (68) that from this equation we can deduce the quantum level mixing given by equation (34). If we make $O_{1}=\widehat{O}, O_{i}=\widehat{I}$ for all $\mathrm{i}=2,3,4 \ldots$ and $m_{1}=0$ in the equation (65) we have

$$
\lim _{n \rightarrow \infty}\left\{(\widehat{\rho}(n) \mid \widehat{O})-(\widehat{\rho}(n) \mid \widehat{I})\left(\widehat{\rho_{*}} \mid \widehat{O}\right)\right\}=0
$$

and since $(\widehat{\rho}(n) \mid \widehat{I})=\operatorname{Tr}(\widehat{\rho}(n))=\operatorname{Tr}(\widehat{\rho}(0))=1$ (conservation of the trace given by equation (21) $)$ we have

$$
\lim _{n \rightarrow \infty}\left\{(\widehat{\rho}(n) \mid \widehat{O})-\left(\widehat{\rho_{*}} \mid \widehat{O}\right)\right\}=0
$$

Then,

$$
\lim _{n \rightarrow \infty}(\widehat{\rho}(n) \mid \widehat{O})=\left(\widehat{\rho_{*}} \mid \widehat{O}\right)
$$

which is identical to the limit

$$
\lim _{t \rightarrow \infty}(\widehat{\rho}(t) \mid \widehat{O})=\left(\widehat{\rho_{*}} \mid \widehat{O}\right)
$$

That is, $\widehat{\rho}(t)$ weakly converges to $\widehat{\rho_{*}}$ corresponding to the mixing case. Therefore, the quantum Kolmogorov level implies the quantum mixing level.

\section{Bernoulli Systems}

Essentially the Bernoulli systems satisfy the mixing conditions but with no $\lim _{t \rightarrow \infty}$ (see e.g. eqs. (7) and (9)). Then these systems satisfy the following equations:

According to paper [1] eq. (BE) the system is Bernoulli if

$$
\mu\left(T_{n} B \cap A\right)=\mu(A) \mu(B)
$$

or

$$
C\left(T_{n} B, A\right)=0
$$

or for the distribution of density function case (see also the corresponding theorem in [19])

$$
\left\langle P_{t} f, g\right\rangle=\left\langle f_{*}, g\right\rangle
$$

or in the quantum case, it is quantum Bernoulli if

$$
(\widehat{\rho}(t) \mid \widehat{O})=\left(\widehat{\rho}_{*} \mid \widehat{O}\right) .
$$

Since the Bernoulli condition (75) is independent of time then it becomes unnecessary to have a discrete version of this condition. 


\subsection{Independent Events}

Let $A \subseteq X$ be an event of the phase space. If we interpret $\mu(A)$ as the probability $P(A)$ of $A$, then Bernoulli systems satisfy a property expressing the independence between two events of the phase space. This property follows directly from its definition. Let $A$ and $B$ be two events, then if $n=0$ in the equation (73) we have the independence events property:

$$
\mu(B \cap A)=\mu(A) \mu(B)
$$

I.e., the probability that $A$ and $B$ occurs simultaneously is the product of the probability of $A$ by the probability of $B$.

Now if we take $\mu_{*}=\mu$ with $\mu_{*}(A)=\int_{A} f_{*}(\phi) d \phi$ then

$$
\begin{aligned}
& \mu_{*}(B \cap A)=\mu_{*}(A) \mu_{*}(B) \\
& \int_{A \cap B} f_{*}(\phi) d \phi=\int_{A} f_{*}(\phi) d \phi \int_{B} f_{*}(\phi) d \phi
\end{aligned}
$$

Namely,

$$
\begin{aligned}
& \int_{X} f_{*} 1_{A} 1_{B} d \phi=\int_{X} f_{*} 1_{A} d \phi \int_{X} f_{*} 1_{B} d \phi \\
& \left\langle f_{*}, 1_{A} 1_{B}\right\rangle=\left\langle f_{*}, 1_{A}\right\rangle\left\langle f_{*}, 1_{B}\right\rangle
\end{aligned}
$$

Let $g_{1}=\sum_{k} a_{k} 1_{A_{k}}$ and $g_{2}=\sum_{l} b_{l} 1_{B_{l}}$ be. From (178) we have

$$
a_{k} b_{l}\left\langle f_{*}, 1_{A_{k}} 1_{B_{l}}\right\rangle=a_{k} b_{l}\left\langle f_{*}, 1_{A_{k}}\right\rangle\left\langle f_{*}, 1_{B_{l}}\right\rangle
$$

By the linearity of the inner product and summing over the indices $\mathrm{k}$ and $\mathrm{l}$ we have

$$
\left\langle f_{*}, \sum_{k} a_{k} 1_{A_{k}} \sum_{l} b_{l} 1_{B_{l}}\right\rangle=\left\langle f_{*}, \sum_{k} a_{k} 1_{A_{k}}\right\rangle\left\langle f_{*}, \sum_{l} b_{l} 1_{B_{l}}\right\rangle
$$

That is,

$$
\left\langle f_{*}, g_{1} g_{2}\right\rangle=\left\langle f_{*}, g_{1}\right\rangle\left\langle f_{*}, g_{2}\right\rangle
$$

Therefore, if $f_{*}=\operatorname{symb}\left(\widehat{\rho_{*}}\right)$ and $g_{1}=\operatorname{symb}\left(\widehat{g_{1}}\right), g_{2}=\operatorname{symb}\left(\widehat{g_{2}}\right)$ where $\widehat{g_{1}}$ and $\widehat{g_{2}}$ are observables we have

$$
\left\langle\operatorname{symb}\left(\widehat{\rho_{*}}\right), g_{1} g_{2}\right\rangle=\left\langle\operatorname{symb}\left(\widehat{\rho_{*}}\right), \operatorname{symb}\left(\widehat{g_{1}}\right)\right\rangle\left\langle\operatorname{symb}\left(\widehat{\rho_{*}}\right), \operatorname{symb}\left(\widehat{g_{2}}\right)\right\rangle
$$

Now from $g_{1}(\phi) g_{2}(\phi) \rightarrow \operatorname{symb}\left(\widehat{g_{1}} \widehat{g_{2}}\right)$ when $\hbar \rightarrow 0$ (see equation (57)) we obtain

$$
\left\langle\operatorname{symb}\left(\widehat{\rho_{*}}\right), \operatorname{symb}\left(\widehat{g_{1}} \widehat{g_{2}}\right)\right\rangle=\left\langle\operatorname{symb}\left(\widehat{\rho_{*}}\right), \operatorname{symb}\left(\widehat{g_{1}}\right)\right\rangle\left\langle\operatorname{symb}\left(\widehat{\rho_{*}}\right), \operatorname{symb}\left(\widehat{g_{2}}\right)\right\rangle
$$

Namely,

$$
\left(\widehat{\rho_{*}} \mid \widehat{g_{1}} \widehat{g_{2}}\right)=\left(\widehat{\rho_{*}} \mid \widehat{g_{1}}\right)\left(\widehat{\rho_{*}} \mid \widehat{g_{2}}\right)
$$

where in (84) we have used the fundamental property of the symb given by the equation (24) of [2]. Moreover, we know that 


$$
\begin{aligned}
& \left(\widehat{\rho}(t) \mid \widehat{g_{1}} \widehat{g_{2}}\right)=\left(\widehat{\rho_{*}} \mid \widehat{g_{1}} \widehat{g_{2}}\right) \\
& \left(\widehat{\rho}(t) \mid \widehat{g_{1}}\right)=\left(\widehat{\rho_{*}} \mid \widehat{g_{1}}\right) \\
& \left(\widehat{\rho}(t) \mid \widehat{g_{2}}\right)=\left(\widehat{\rho_{*}} \mid \widehat{g_{2}}\right)
\end{aligned}
$$

Therefore we can express (84) as

$$
\left(\widehat{\rho}(t) \mid \widehat{g_{1}} \widehat{g_{2}}\right)=\left(\widehat{\rho}(t) \mid \widehat{g_{1}}\right)\left(\widehat{\rho}(t) \mid \widehat{g_{2}}\right)
$$

for all pairwise of observables, $\widehat{g_{1}} \widehat{g_{2}}$. If we generalize for an arbitrary product of observables from (86) we have that

$$
\left(\widehat{\rho}(t) \mid \prod_{i} \widehat{g}_{i}\right)=\prod_{i}\left(\widehat{\rho}(t) \mid \widehat{g_{i}}\right)
$$

The equation (87) is the translation into quantum language of the independence of events expressed by the equation (76). Physically, it tells us that, in the classical limit of a Bernoulli system the mean value of an arbitrary product of observables factorizes into the product of the mean values of each observable and this factorization occurs at all times, and this quantum factorization express the no-correlation of the observables $\widehat{g_{1}}, \widehat{g_{2}}, .$. of $(87)$ in a Bernouilli system.

\subsection{Particular Case: Kolmogorov}

Bernoulli level is included in Kolmogorov level (equation (73)). This fact implies equation (39) so this property must be verified by the respective quantum versions on these levels. We consider a numerable set of observables $\widehat{O}_{1}, \widehat{O}_{2}, \widehat{O}_{3}, \ldots$ and a sequence $m_{1}, m_{2}, m_{3} \ldots \in \mathbb{N}_{0}$. To demonstrate that quantum Bernoulli level implies quantum Kolmogorov level we use the quantum version of the independence events property given by the equation (87). If we call $\widehat{g}_{1}=\widehat{O}_{1}, \widehat{g}_{2}=$ $\prod_{j=2}^{\infty} \widehat{O}_{j}\left(n+m_{j}\right)$ for all $j=2,3,4, \ldots$ by the equation (87) we have

$$
\left(\widehat{\rho}\left(n+m_{1}\right) \mid \widehat{O}_{1} \prod_{j=2}^{\infty} \widehat{O}_{j}\left(n+m_{j}\right)\right)=\left(\widehat{\rho}\left(n+m_{1}\right) \mid \widehat{O}_{1}\right) \prod_{j=2}^{\infty}\left(\widehat{\rho}\left(n+m_{1}\right) \mid \widehat{O}_{j}\left(n+m_{j}\right)\right)
$$

In particular since the system is Bernoulli we have

$$
\left(\widehat{\rho}\left(n+m_{1}\right) \mid \widehat{O}_{1}\right)=\left(\widehat{\rho}_{*} \mid \widehat{O}_{1}\right)
$$

From equations (88) and (89) we have that

$$
\left(\widehat{\rho}\left(n+m_{1}\right) \mid \widehat{O}_{1} \prod_{j=2}^{\infty} \widehat{O}_{j}\left(n+m_{j}\right)\right)=\prod_{j=2}^{\infty}\left(\widehat{\rho}\left(n+m_{1}\right) \mid \widehat{O}_{j}\left(n+m_{j}\right)\right)\left(\widehat{\rho}_{*} \mid \widehat{O}_{1}\right)
$$

Therefore,

$$
\lim _{n \rightarrow \infty}\left(\widehat{\rho}\left(n+m_{1}\right) \mid \widehat{O}_{1} \prod_{j=2}^{\infty} \widehat{O}_{j}\left(n+m_{j}\right)\right)=\lim _{n \rightarrow \infty} \prod_{j=2}^{\infty}\left(\widehat{\rho}\left(n+m_{1}\right) \mid \widehat{O}_{j}\left(n+m_{j}\right)\right)\left(\widehat{\rho}_{*} \mid \widehat{O}_{1}\right)
$$


That is,

$$
\lim _{n \rightarrow \infty}\left\{\left(\widehat{\rho}\left(n+m_{1}\right) \mid \widehat{O}_{1} \prod_{j=2}^{\infty} \widehat{O}_{j}\left(n+m_{j}\right)\right)-\prod_{j=2}^{\infty}\left(\widehat{\rho}\left(n+m_{1}\right) \mid \widehat{O}_{j}\left(n+m_{j}\right)\right)\left(\widehat{\rho}_{*} \mid \widehat{O}_{1}\right)\right\}=0
$$

which is the quantum Kolmogorov condition (see equation (68)).

\section{Physical Relevance of QEH: Casati-Prosen model and Kicked Rotator}

In this section we give a physical relevance to the Quantum Ergodic Hierarchy analyzing in terms of the QEH levels two standard models of the quantum chaos literature: the CasatiProsen model [4] and the kicked rotator $([6],[7],[8])$. These models are emblematic for quantum chaos because the first one contains the mean features of the chaotic billiards which are one of the pioneers experiments made in the field $([6,7])$ and the two others contains the physics of many relevant phenomenons (see [6] chapter 4.2) like the Anderson localization, the hydrogen atom in an electric field, etc.

\subsection{The Casati-Prosen model in the quasi-continuous spectrum approxima- tion}

Casati-Prosen model is a Sinai billiard provided with a quantum paradigmatic phenomenon the double slit experiment. On the other hand the classical Sinai billiards are a wellknown type of Kolmogorov systems and therefore they are mixing systems. In this subsection we review the quasi-continuous spectrum approximation we used in [5] to explain the Casati-Prosen model conceptually. We briefly begin summarizing these arguments:

- For large times but shorter than the time $t^{*}=\frac{2 \pi \hbar}{\min \left|E_{i}-E_{i+1}\right|}$ of the quantum system, that is $t \ll t^{*}$, we can assume that the energy spectrum is quasi continuous and therefore we can replace sums by integrals in all the equations. At this point we consider the system is mixing (moreover it is a K-system) and then due to the decoherence of the Sinai billiard the interference is calculated in the equilibrium state $\widehat{\rho}_{*}$ which is the weak limit of the initial state $\widehat{\rho}(0)$ (mixing condition).

- To define the nonintegrability of the Sinai billiard we use the local CSCO of [2] $\left\{\widehat{H}, \widehat{O}_{\phi_{i}}\right\}$. For this case $\widehat{O}_{\phi_{i}}=\widehat{P}_{\phi_{i}}$ is the local momentum and $\widehat{P}_{\phi_{i}}=\left(\widehat{P}_{x}, \widehat{P}_{y}\right)_{\phi_{i}}$.

\footnotetext{
${ }^{5}$ Strictly, $t^{*}$ is an approximation to the Poincare time 24 since $e^{-i\left(\frac{E_{n}-E_{m}}{\hbar}\right) t^{*}}=e^{-2 \pi i \frac{E_{n}-E_{m}}{m i n\left|E_{i}-E_{i+1}\right|}} \approx 1$ if and only if $\frac{E_{n}-E_{m}}{\min \left|E_{i}-E_{i+1}\right|}$ is an integer for all $n, m$ but in general this requirement can be fulfilled for some initial conditions $\rho(0)$. To avoid these problems we indeed should use $\max \left|E_{i}-E_{i+1}\right|$ instead of $\min \left|E_{i}-E_{i+1}\right|$ for the quasi-continuous approximation. If we put $t^{*}=\frac{2 \pi \hbar}{\max \left|E_{i}-E_{i+1}\right|}$ since $\max \left\{\left|E_{i}-E_{i+1}\right|\right\} \geq \Delta E$ where $\Delta E$ is mean energy level spacing it follows that $t^{*} \leq t_{H}$ where $t_{H}=\frac{2 \pi \hbar}{\Delta E}$ is the Heisenberg time [25]. This hypothesis is reasonable because quantum chaos phenomena with a semiclassical description such as relaxation, exponential sensitivity etc. are possible within a time scale $t \leq t^{*}$. Then, the quasi continuous spectrum approximation is obtained as follows: if $t \ll t^{*}$ for all finite times $t \Longrightarrow t^{*}=\frac{2 \pi \hbar}{\max \left|E_{i}-E_{i+1}\right|} \rightarrow \infty \Longrightarrow \frac{\max \left|E_{i}-E_{i+1}\right|}{2 \pi \hbar} \approx 0 \Longrightarrow$ $\frac{\left|E_{i}-E_{i+1}\right|}{2 \pi \hbar} \approx 0$ then $\frac{E_{i}-E_{i+1}}{\hbar}$ is infinitesimal. Therefore, we can replace any sum $\sum_{j} f\left(E_{j}\right) e^{-i \frac{E_{j}}{\hbar} t}$ by the integral $\int d E f(E) e^{-i \frac{E}{\hbar} t}$. Then when $t \ll t^{*}$ the Riemann-Lebesgue theorem can be used.
} 
- In these terms, the interference $P_{\text {int }}$ of the state $\rho(t)=|\varphi(x, t)\rangle\langle\varphi(x, t)|$ (where $|\varphi(x, t)\rangle=$ $\left|\varphi_{1}(x, t)\right\rangle+\left|\varphi_{2}(x, t)\right\rangle$ and $\left|\varphi_{i}(x, t)\right\rangle$ are the two circular-symmetric solutions produced by the boundary conditions in the two slits as a result of the direct impact of the initial gaussian wavepacket) it is given by $2 \operatorname{Re}\left(\varphi_{1}(x, t) \varphi_{2}(x, t)^{*}\right)$.

- The state $\rho(t)=|\varphi(x, t)\rangle\langle\varphi(x, t)|$ is replaced by the equilibrium state $\widehat{\rho}_{*}$ where the time dependence has disappeared in the equilibrium. As a consequence of the linearity of the Schrodinger equation and by the local CSCO $\left\{\widehat{H}, \widehat{P}_{\phi_{i}}\right\}$ the interference is $P_{i n t}=$ $2 \sum_{i p w} \rho(w)_{\phi_{i}, p} \operatorname{Re}\left(\varphi_{1 w p}(x) \varphi_{2 w p}(x)^{*}\right)$.

- To compute $P_{i n t}$ we use the unitary transform $U_{p \phi}^{m}$ that diagonalizes $\left(w, m,\left.m^{\prime}\right|_{\phi}\right.$ so $P_{\text {int }}$ is

$$
P_{\text {int }}=\sum_{i, p, p^{\prime}, w, m, m^{\prime}} \rho(w)_{\phi_{i}, p, p^{\prime}}\left[U_{p \phi_{i}}^{m}\left(U_{p^{\prime} \phi_{i}}^{m^{\prime}}\right)^{*} e^{-\frac{i}{\hbar}\left(\mathbf{m}-\mathbf{m}^{\prime}\right) \cdot \mathbf{x}} e^{-\frac{i}{\hbar}\left(\frac{\mathbf{m}+\mathbf{m}^{\prime}}{2}\right) \cdot \mathbf{s}}+C . C .\right]
$$

where C.C. denotes the complex conjugate and $\mathbf{s}=(s, 0)$ where $s$ is the distance between the slits. The set $\{w\}$ is the energy spectrum which we assume quasi-continuous and $\mathbf{m}=\hbar \mathbf{k}$ labels each wave vector in the expansion.

- Now because there is a macroscopic distance from the two slits screen to the photographic plate, $\mathbf{x}$ is macroscopic with respect to $\hbar$ in such a way that we can consider that $\frac{x}{\hbar} \rightarrow \infty$ and we can use the Riemann-Lebesgue theorem concluding that $P_{\text {int }}=0$.

In this way in [5] we demonstrate that the interference fringes vanish due to the decoherence in the equilibrium state. From the viewpoint of the Quantum Ergodic Hierarchy the equilibrium state $\widehat{\rho}_{*}$ of the Casati model is a consequence that this model belongs to the mixing level. And in turn under the hypothesis mentioned above the mixing of Casati-Prosen model implies the cancellation of the interference fringes. On the other hand, Casati explains his model [4] by a computer experiment that shows how complexity can produce this decoherence. Therefore the relevant observation is that the Quantum Ergodic Hierarchy brings us a conceptual framework where computability can be tested and moreover, this test is in agreement with the theoretical results of QEH [5]. This is a proof of the physical relevance to QEH.

\subsection{The Explanation Of Casati-Prosen model in terms of the ergodic level}

From all above arguments mentioned in the section 7.1 two "natural" objections can be made: Since most of chaotic systems of interest are bounded systems with energy spectrum discrete, can we be sure that hypothesis quasi-continuous spectrum is valid for all these systems? And also, since in all expressions of the QEH levels the time goes to infinity, What happens when the time $t^{*}=\frac{2 \pi \hbar}{\min \left|E_{i}-E_{i+1}\right|}$ is small?

The answer to the first question is negative, and in such a case we can still explain these systems in terms of QEH. In the next subsection and below we show this fact and also this is the answer to the second objection. Therefore, we end this subsection giving an alternative

\footnotetext{
${ }^{6}$ Note: $\mathbf{x}$ and $\hbar$ have not the same units and therefore the limit $\frac{x}{\hbar} \rightarrow \infty$ has the following explanation. Since $\mathbf{m}=\hbar \mathbf{k}$ the factor $e^{-\frac{i}{\hbar}\left(\mathbf{m}-\mathbf{m}^{\prime}\right) \cdot \mathbf{x}}$ in (93) is equal to $e^{-i\left(\mathbf{k}-\mathbf{k}^{\prime}\right) \cdot \mathbf{x}}$. Further, because $E=\frac{\hbar \mathbf{k}^{2}}{2 M}$ in the quasicontinuous spectrum approximation, $\mathbf{k}$ is quasi-continuous. Moreover, since $|\mathbf{k}|=\frac{2 \pi}{\lambda}$ and $\mathbf{x}$ is macroscopic with respect to $\lambda$ (for example, in a electron wavepacket $\lambda$ is typically of the order of $10^{-13} \mathrm{~cm}$ ) we can consider that $\frac{2 \pi \mathbf{x}}{\lambda}=\mathbf{k} \cdot \mathbf{x} \rightarrow \infty$. Now we can we can use the Riemann-Lebesgue theorem in the sum of eq. (93) concluding that $P_{\text {int }}=0$.
} 
explanation. That is, we strictly assume that the energy spectrum of the Casati-Prosen model is discrete. This is so because the Casati-Prosen model is a bounded system and therefore the energy spectrum is discrete.

First, due to that the Casati-Prosen model is a Kolmogorov then it is ergodic. In particular, the equilibrium state $\widehat{\rho}_{*}$ is the Cèsaro limit of the initial Gaussian wavepacket $\widehat{\rho}(0)$. On the other hand the initial state written in terms of the local CSCO $\left\{\widehat{H}, \widehat{P}_{\phi_{i}}\right\}$ is given by [2]

$$
\widehat{\rho}(0)=\sum_{i, p, p^{\prime}, w_{\alpha}, w_{\alpha}^{\prime}} \rho_{w_{\alpha}, w_{\alpha}^{\prime}, \phi_{i}, p, p^{\prime}} U_{p \phi_{i}}^{m}\left(U_{p^{\prime} \phi_{i}}^{m^{\prime}}\right)^{*}\left|w_{\alpha}, m\right\rangle_{\phi_{i}}\left\langle w_{\alpha}^{\prime},\left.m^{\prime}\right|_{\phi_{i}}\right.
$$

Then the state at the time $t$ is

$$
\widehat{\rho}(t)=\sum_{i, p, p^{\prime}, w_{\alpha}, w_{\alpha}^{\prime}} \rho_{w_{\alpha}, w_{\alpha}^{\prime}, \phi_{i}, p, p^{\prime}} U_{p \phi_{i}}^{m}\left(U_{p^{\prime} \phi_{i}}^{m^{\prime}}\right)^{*} e^{-\frac{i}{\hbar}\left(w_{\alpha}-w_{\alpha}^{\prime}\right) t}\left|w_{\alpha}, m\right\rangle_{\phi_{i}}\left\langle w_{\alpha}^{\prime},\left.m^{\prime}\right|_{\phi_{i}}\right.
$$

We are interested in the probability amplitude $|\varphi(\mathbf{x}, t)|^{2}$ (see eq. (6) of [5]) at $\mathbf{x}$ for the state $\widehat{\rho}(t)$ in the limit $t \rightarrow \infty$. Then this amplitude is given by

$$
\left\langle\mathbf{x}|\widehat{\rho}(t)| \mathbf{x}^{\prime}\right\rangle=\sum_{i, p, p^{\prime}, w_{\alpha}, w_{\alpha}^{\prime}} \rho_{w_{\alpha}, w_{\alpha}^{\prime}, \phi_{i}, p, p^{\prime}} U_{p \phi_{i}}^{m}\left(U_{p^{\prime} \phi_{i}}^{m^{\prime}}\right)^{*} e^{-\frac{i}{\hbar}\left(w_{\alpha}-w_{\alpha}^{\prime}\right) t}\left\langle\mathbf{x} \mid w_{\alpha}, m\right\rangle_{\phi_{i}}\left\langle w_{\alpha}^{\prime}, m^{\prime} \mid \mathbf{x}^{\prime}\right\rangle_{\phi_{i}}
$$

where again as in the paper [5] we have the replacements $\mathbf{x} \longleftrightarrow \mathbf{x}-\frac{1}{2} \mathbf{s}$ and $\mathbf{x} \longleftrightarrow \mathbf{x}^{\prime}+\frac{1}{2} \mathbf{s}$ and also

$$
\begin{aligned}
\left\langle\mathbf{x} \mid w_{\alpha}, m\right\rangle_{\phi_{i}} & \sim e^{-\frac{i}{\hbar}(\mathbf{m} \cdot \mathbf{x})} \\
\left\langle w_{\alpha}^{\prime}, m^{\prime} \mid \mathbf{x}^{\prime}\right\rangle_{\phi_{i}} & \sim e^{\frac{i}{\hbar}\left(\mathbf{m}^{\prime} \cdot \mathbf{x}^{\prime}\right)}
\end{aligned}
$$

From these two last equations (96) and (97) we have

$$
\langle\mathbf{x}|\widehat{\rho}(t)| \mathbf{x}\rangle=\sum_{i, p, p^{\prime}, w_{\alpha}, w_{\alpha}^{\prime}} \rho_{w_{\alpha}, w_{\alpha}^{\prime}, \phi_{i}, p, p^{\prime}} U_{p \phi_{i}}^{m}\left(U_{p^{\prime} \phi_{i}}^{m^{\prime}}\right)^{*} e^{-\frac{i}{\hbar}\left(\mathbf{m}-\mathbf{m}^{\prime}\right) \cdot \mathbf{x}} e^{\frac{i}{2 \hbar}\left(\mathbf{m}+\mathbf{m}^{\prime}\right) \cdot \mathbf{s}} e^{-\frac{i}{\hbar}\left(w_{\alpha}-w_{\alpha}^{\prime}\right) t}
$$

for the probability amplitude. In order to have an explanation in terms of the ergodic level we must interpret this amplitude as the mean value of some observable $\widehat{O}$, calculated in the state $\widehat{\rho}(t)$. If we choose $\widehat{O}=|\mathbf{x}\rangle\langle\mathbf{x}|$ then we have that

$$
\langle\mathbf{x}|\widehat{\rho}(t)| \mathbf{x}\rangle=\langle\mid \mathbf{x}\rangle\langle\mathbf{x} \mid\rangle_{\widehat{\rho}(t)}
$$

This means that the probability amplitude $\langle\mathbf{x}|\widehat{\rho}(t)| \mathbf{x}\rangle$ is the mean value of the projector $|\mathbf{x}\rangle\langle\mathbf{x}|$ in the state $\widehat{\rho}(t)$. Now since the system is ergodic (eq. (29), (301)) we expect that the time average of this amplitude is equal to the mean value of $|\mathbf{x}\rangle\langle\mathbf{x}|$ in the state $\widehat{\rho}_{*}$ in the limit $t \rightarrow \infty$. More precisely, if we use the continuous version

\footnotetext{
${ }^{7}$ Since in this case we have a continuous evolution given by the evolution operator $\widehat{U}(t)$ we can choose any of the two versions of the ergodic level (eq. (29), (30) ). Both give the same result.
} 


$$
\left.\lim _{T \rightarrow \infty} \frac{1}{T} \int_{0}^{T}\langle\mathbf{x}|\widehat{\rho}(t)| \mathbf{x}\rangle d t=\left(\widehat{\rho}_{*}|| \mathbf{x}\right\rangle\langle\mathbf{x}|\right)=\text { cte. }
$$

This equation means that in the Casati-Prosen model the amplitude tends in time average to the constant $\left.\left(\widehat{\rho}_{*}|| \mathbf{x}\right\rangle\langle\mathbf{x}|\right)$ and therefore that the average of the interference term $P_{\text {int }}$ vanishes in time. Now we calculate the average value of $\langle\mathbf{x}|\widehat{\rho}(t)| \mathbf{x}\rangle$. First, from (98) we can separate the sum of $\langle\mathbf{x}|\widehat{\rho}(t)| \mathbf{x}\rangle$ in a diagonal and non-diagonal terms where the non-diagonal term is the interference term $P_{\text {int }}$. Then

$$
\langle\mathbf{x}|\widehat{\rho}(t)| \mathbf{x}\rangle=P_{\text {diag }}+P_{\text {int }}
$$

where

$$
\begin{aligned}
& P_{\text {diag }}=\sum_{i, p, w_{\alpha}} \rho_{w_{\alpha}, \phi_{i}, p}\left|U_{p \phi_{i}}^{m}\right|^{2} e^{\frac{i}{\hbar} \mathbf{m} \cdot \mathbf{s}}=c t e . \\
& P_{\text {int }}=\sum_{i, p, p^{\prime}, w_{\alpha} \neq w_{\alpha}^{\prime}} \rho_{w_{\alpha}, w_{\alpha}^{\prime}, \phi_{i}, p, p^{\prime}} U_{p \phi_{i}}^{m}\left(U_{p^{\prime} \phi_{i}}^{m^{\prime}}\right)^{*} e^{-\frac{i}{\hbar}\left(\mathbf{m}-\mathbf{m}^{\prime}\right) \cdot \mathbf{x}} e^{\frac{i}{2 \hbar}\left(\mathbf{m}+\mathbf{m}^{\prime}\right) \cdot \mathbf{s}} e^{-\frac{i}{\hbar}\left(w_{\alpha}-w_{\alpha}^{\prime}\right) t}
\end{aligned}
$$

In the non-diagonal term $P_{\text {int }}$ of the equation (102) we have assumed the crucial hypothesis of non-degeneracy $w_{\alpha} \neq w_{\alpha}^{\prime}$ which is one of the mean features of the chaotic billiards, e.g. the GOE, GUE and GSE spectral distributions (see for example 6], 7], 8]). The nondegeneracy is a necessary condition for quantum chaos in the billiard systems. Therefore, since the Casati-Prosen model is a chaotic billiard then, in (102) we cannot have terms of the type $\sum_{i, p, p^{\prime}, w_{\alpha}} \rho_{w_{\alpha}, \phi_{i}, p, p^{\prime}} U_{p \phi_{i}}^{m}\left(U_{p^{\prime} \phi_{i}}^{m^{\prime}}\right)^{*} e^{-\frac{i}{\hbar}\left(\mathbf{m}-\mathbf{m}^{\prime}\right) \cdot \mathbf{x}} e^{\frac{i}{2 \hbar}\left(\mathbf{m}+\mathbf{m}^{\prime}\right) \cdot \mathbf{s}}$. Then it is enough to show that $\lim _{T \rightarrow \infty} \frac{1}{T} \int_{0}^{T} P_{\text {int }}(\mathbf{x}, t) d t=0$. We have

$$
\begin{aligned}
& \lim _{T \rightarrow \infty} \frac{1}{T} \int_{0}^{T}\langle\mathbf{x}|\widehat{\rho}(t)| \mathbf{x}\rangle d t=\lim _{T \rightarrow \infty} \frac{1}{T} \int_{0}^{T} P_{\text {diag }} d t+\lim _{T \rightarrow \infty} \frac{1}{T} \int_{0}^{T} P_{\text {int }}(\mathbf{x}, t) d t= \\
& =P_{\text {diag }}+\lim _{T \rightarrow \infty} \frac{1}{T} \int_{0}^{T} P_{\text {int }}(\mathbf{x}, t) d t= \\
& =P_{\text {diag }}+ \\
& +\sum_{i, p, p^{\prime}, w_{\alpha} \neq w_{\alpha}^{\prime}} \rho_{w_{\alpha}, w_{\alpha}^{\prime}, \phi_{i}, p, p^{\prime}} U_{p \phi_{i}}^{m}\left(U_{p^{\prime} \phi_{i}}^{m^{\prime}}\right)^{*} e^{-\frac{i}{\hbar}\left(\mathbf{m}-\mathbf{m}^{\prime}\right) \cdot \mathbf{x}} e^{\frac{i}{2 \hbar}\left(\mathbf{m}+\mathbf{m}^{\prime}\right) \cdot \mathbf{s}} l i m_{T \rightarrow \infty} \frac{1}{T} \int_{0}^{T} e^{-\frac{i}{\hbar}\left(w_{\alpha}-w_{\alpha}^{\prime}\right) t} d t= \\
& =P_{\text {diag }}+ \\
& +\sum_{i, p, p^{\prime}, w_{\alpha} \neq w_{\alpha}^{\prime}} \rho_{w_{\alpha}, w_{\alpha}^{\prime}, \phi_{i}, p, p^{\prime}} U_{p \phi_{i}}^{m}\left(U_{p^{\prime} \phi_{i}}^{m^{\prime}}\right)^{*} e^{-\frac{i}{\hbar}\left(\mathbf{m}-\mathbf{m}^{\prime}\right) \cdot \mathbf{x}} e^{\frac{i}{2 \hbar}\left(\mathbf{m}+\mathbf{m}^{\prime}\right) \cdot \mathbf{s}} l i m_{T \rightarrow \infty} \frac{i \hbar\left(e^{-\frac{i}{\hbar}\left(w_{\alpha}-w_{\alpha}^{\prime}\right) T}-1\right)}{T\left(w_{\alpha}-w_{\alpha}^{\prime}\right)} \\
& \left.=P_{\text {diag }}=\sum_{i, p, w_{\alpha}} \rho_{w_{\alpha}, \phi_{i}, p}\left|U_{p \phi_{i}}^{m}\right|^{2} e^{\frac{i}{\hbar} \mathbf{m} \cdot \mathbf{s}}=\left(\widehat{\rho}_{*}|| \mathbf{x}\right\rangle\langle\mathbf{x}|\right)=c t e .
\end{aligned}
$$

Then $\lim _{T \rightarrow \infty} \frac{1}{T} \int_{0}^{T} P_{\text {int }}(\mathbf{x}, t) d t=0$ and $\widehat{\rho}_{*}=\sum_{i, p, w_{\alpha}} \rho_{w_{\alpha}, \phi_{i}, p}\left|U_{p \phi_{i}}^{m}\right|^{2}\left|w_{\alpha}, m\right\rangle_{\phi_{i}}\left\langle w_{\alpha},\left.m\right|_{\phi_{i}}\right.$. 
We have obtained $\widehat{\rho}_{*}$ the Cèsaro limit of the initial Gaussian wavepacket $\widehat{\rho}(0)$. Therefore we show that without doing any kind of hypothesis about the energy spectrum, the equilibrium limit of Casati-Prosen model is the Cèsaro limit $\widehat{\rho}_{*}$ and as a consequence the interference fringes vanish "on time average" for $t \rightarrow \infty$. This is physically expected since the human eye averages in a scale time which is extremely larger than the characteristic times of the decoherence of the quantum systems and therefore we see that "on time average" the interference fringes vanish. This is the content of the ergodic level for the Casati-Prosen model without any hypothesis about the energy spectrum.

Summarizing, the behavior of the Casati-Prosen model we can conceptually explain, in the mentioned two ways, quasi-continuous approximation and ergodic level. The first explanation is possible because its energy spectrum that is discrete can be approximated by a quasi-continuous one. The two explanations are not in contradictory but both differ in the type of decoherence. In the quasi-continuous case it can be demonstrated that the interference fringes vanish while using the ergodic level we strictly can demonstrate that these vanish on time average. On the other hand, when the quasi-continuous approximation is not valid then we can always use the ergodic level. We remark the satisfying aspect is that both explanations are based on the first two levels of the Quantum Ergodic Hierarchy, mixing and ergodic respectively.

\subsection{The Kicked Rotator}

For the reasons that we have mentioned at the beginning the kicked rotator is one of most famous and studied chaotic systems. The kicked rotator expresses in a simple way the physics of chaotic systems whose Hamiltonian are of the type $H_{0}+H^{\prime}$ where $H_{0}$ is the unperturbed and integrable Hamiltonian and $H^{\prime}$ is a delta time periodic perturbation. This is important since the dynamics of many quantum systems which presents chaos can be mapped into the kicked rotator. The Hamiltonian is given by (see [6] eq. 4.2.1)

$$
H=\widehat{L}^{2}+\lambda \cos \widehat{\theta} \sum_{n} \delta(t-n)
$$

which it describes the free rotation of a pendulum with angular momentum $\widehat{L}$ periodically kicked by a gravitational potential of strength $\lambda$. The moment of inertia $I$ and the kick period $T$ are normalized to one. Classically, the kicked rotator presents different behaviors for several values of the parameter $\lambda$. For small $\lambda$ values the rotator shows regular behavior for most initial values of $\theta$ and $L$ with integrable regions of the space phase. But with increasing $\lambda$ the phase space becomes more and more chaotic, until for $\lambda>5$ most regular parts have disappeared (see Fig. 1.3.(c) of [6]).

For a quantum mechanical description we need the evolution operator which is given by the Floquet operator $\widehat{F}$ (see [6] eq. 4.2.12)

$$
\widehat{F}=e^{\frac{-i}{\hbar} \lambda \cos \widehat{\theta}} e^{\frac{-i}{2 \hbar} \tau \widehat{L}^{2}}
$$

We note that since this evolution is discrete then we must use the corresponding discrete versions of the levels of QEH. We can express any initial state $\rho(0)$ in the eigenbasis of $\widehat{L},|n\rangle=\frac{1}{\sqrt{2 \pi}} e^{i n \theta}$

$$
\rho(0)=\sum_{n} a_{n}|n\rangle\left\langle n\left|+\sum_{n \neq m} a_{n m}\right| n\right\rangle\langle m|
$$

In order to obtain the Césaro weak limit of $\widehat{\rho}(t)$ it is more convenient to express the initial state of (106) in the eigenbasis $\{|k\rangle\}$ of $\widehat{F}$. 


$$
\rho(0)=\sum_{k} \rho_{k k}|k\rangle\left\langle k\left|+\sum_{k \neq k^{\prime}} \rho_{k k^{\prime}}\right| k\right\rangle\left\langle k^{\prime}\right|
$$

Now we show that $\widehat{\rho}_{*}=\sum_{k} \rho_{k k}|k\rangle\langle k|$ is the Cèsaro limit of $\widehat{\rho}(t)$ for all value of the parameter $\lambda$ and therefore, the kicked rotator belongs to the ergodic level of the QEH for all $\lambda$.

$N$ successive applications of $\widehat{F}$ to $\widehat{\rho}(0)$ give the state $\widehat{\rho}$ at the instant of time $t=N \tau$. More precisely, we have

$$
\widehat{\rho}(N \tau)=\sum_{k} \rho_{k k}|k\rangle\left\langle k\left|+\sum_{k \neq k^{\prime}} \rho_{k k^{\prime}} e^{-i N\left(\phi_{k}-\phi_{k^{\prime}}\right)}\right| k\right\rangle\left\langle k^{\prime}\right|
$$

where the first and the second sums of (108) are the diagonal and non-diagonal terms of the state $\widehat{\rho}(N \tau)$, and the phase $e^{-i N \phi_{k}}$ is the eigenvalue of the eigenstate $|k\rangle$ (see [6] page 136 and 137). Let $\widehat{O}$ be an observable. From (108) the mean value of $\widehat{O}$ in the state $\widehat{\rho}(N \tau)$ is

$$
\langle\widehat{O}\rangle_{\widehat{\rho}(N \tau)}=(\widehat{\rho}(N \tau) \mid \widehat{O})=\operatorname{Tr}(\widehat{\rho}(N \tau) \widehat{O})=\sum_{k} \rho_{k k} O_{k k}+\sum_{k \neq k^{\prime}} \rho_{k k^{\prime}} e^{-i N\left(\phi_{k}-\phi_{k^{\prime}}\right)} O_{k k^{\prime}}
$$

The second sum of (109) are the interference terms, proper of the quantum mechanical phenomena and the cancellation of this term is an expression of decoherence. From (108) we have

$$
\begin{aligned}
& \lim _{N \rightarrow \infty} \frac{1}{N} \sum_{j=0}^{N-1}(\widehat{\rho}(j \tau) \mid \widehat{O})=\lim _{N \rightarrow \infty} \frac{1}{N} \sum_{j=0}^{N-1}\left\{\sum_{k} \rho_{k k} O_{k k}+\sum_{k \neq k^{\prime}} \rho_{k k^{\prime}} e^{-i j\left(\phi_{k}-\phi_{k^{\prime}}\right)} O_{k k^{\prime}}\right\}= \\
& =\sum_{k} \rho_{k k} O_{k k}+\sum_{k \neq k^{\prime}} \rho_{k k^{\prime}} O_{k k^{\prime}} \lim _{N \rightarrow \infty} \frac{1}{N}\left\{\sum_{j=0}^{N-1}\left(e^{-i\left(\phi_{k}-\phi_{k^{\prime}}\right)}\right)^{j}\right\}= \\
& =\sum_{k} \rho_{k k} O_{k k}+\sum_{k \neq k^{\prime}} \rho_{k k^{\prime}} O_{k k^{\prime}} \lim _{N \rightarrow \infty} \frac{1-e^{-i N\left(\phi_{k}-\phi_{k^{\prime}}\right)}}{N\left(1-e^{-i\left(\phi_{k}-\phi_{k^{\prime}}\right)}\right)}=\sum_{k} \rho_{k k} O_{k k}=\left(\widehat{\rho}_{*} \mid \widehat{O}\right)
\end{aligned}
$$

where we have used that $\lim _{N \rightarrow \infty} \frac{1-e^{-i N\left(\phi_{k}-\phi_{k^{\prime}}\right)}}{N\left(1-e^{-i\left(\phi_{k}-\phi_{k^{\prime}}\right)}\right)}=0$ for all $k, k^{\prime}$ and that $\widehat{\rho}_{*}=\sum_{k} \rho_{k k}|k\rangle\langle k|$. From (110) and the discrete version of the ergodic level (30) the kicked rotator is ergodic for all $\lambda$ and the Cèsaro limit is $\widehat{\rho}_{*}=\sum_{k} \rho_{k k}|k\rangle\langle k|$ which is the equilibrium state "on time-average". In this time-average sense we can say that the kicked rotator decoheres to the state $\sum_{k} \rho_{k k}|k\rangle\langle k|$ for all initial state and the decoherence time is $\infty$.

Now we see that for values of $\lambda>5$ the behavior is different from the ergodic case. In such case the expected quantum mechanically distribution $f_{N}(L)$ for the quadratic mean value of the angular momentum $\left\langle\widehat{L}^{2}\right\rangle$ after $N$ kicks is given by (see [6] eq. 4.2.20)

$$
f_{N}(L)=\frac{1}{l_{s}} e^{-\frac{2|L|}{l_{s}}}
$$


This exponential localization implies that for kick numbers $N \leq l_{s}$ we are in the range of classical diffusion and for $N \gg l_{s}$ we are in the fully chaotic behavior 8 . For $N \gg l_{s}$ the phase factors $e^{-i N\left(\phi_{k}-\phi_{k^{\prime}}\right)}$ of (109) oscillate rapidly in such a way that only survive the terms with $k=k^{\prime}$, that is

$$
\langle\widehat{O}\rangle_{\widehat{\rho}(N \tau)}=(\widehat{\rho}(N \tau) \mid \widehat{O}) \simeq \sum_{k} \rho_{k k} O_{k k}=\left(\widehat{\rho}_{*} \mid \widehat{O}\right) \quad \text { for } N \gg l_{s}
$$

where $\widehat{\rho}_{*}=\sum_{k} \rho_{k k}|k\rangle\langle k|$. Then from (112) in the case $\lambda>5$ we have

$$
\lim _{N \rightarrow \infty}(\widehat{\rho}(N) \mid \widehat{O})=\lim _{N \rightarrow \infty}(\widehat{\rho}(N \tau) \mid \widehat{O})=\sum_{k} \rho_{k k} O_{k k}=\left(\widehat{\rho}_{*} \mid \widehat{O}\right)
$$

Now by the mixing condition (eq.(35) ) for the discrete case, the equation (113) says that for $\lambda>5$ the kicked rotator belongs to the mixing level and the equilibrium state is the weak limit $\widehat{\rho}_{*}=\sum_{k} \rho_{k k}|k\rangle\langle k| . \widehat{\rho}_{*}$ is the diagonal part of the initial state $\widehat{\rho}(0)$ (see eq. (107)) written in the basis of the Floquet operator $\widehat{F}$. This situation is analogue to SID [26] where the decoherence is performed in the energy basis. Meanwhile, for the fully chaotic regime $10>5$ the kicked rotator decoheres in the Floquet basis $\{|k\rangle\}$.

Moreover, from equation (112) and the Bernoulli condition (75) we have $(\widehat{\rho}(t) \mid \widehat{O})=\sum_{k} \rho_{k k} O_{k k}$ $=\left(\widehat{\rho}_{*} \mid \widehat{O}\right)$ for $t=N \tau \gg \tau l_{s}$. That is, the kicked rotator is Bernoulli from any time $t \gg \tau l_{s}$. And this characteristic time $t_{D}=\tau l_{s}$ expresses the decoherence of the kicked rotator. In this way we conclude the kicked rotator analysis in terms of the Quantum Ergodic Hierarchy.

\section{Conclusions}

In this paper we have introduced a definition of the four main levels of the Quantum Ergodic Hierarchy with the property that their classical limits are the corresponding usual levels of the classical ergodic hierarchy.

Language translation of the sigma algebras of the Kolmogorov level to quantum language could be made and reduced to a single condition (see equation (68) ) thanks to the application of the principle of inclusion-exclusion (see equation (43)), which helped us to extend the technique used in the paper [2] for the ergodic level and mixing level. Here we have used the properties of the Wigner transform. Thus the resulting condition for the Kolmogorov quantum level is consistent with the definitions of mixing and Bernoulli (see 5.2 and 6.2 sections).

Language translation of the Bernoulli level was the most immediate of all levels of the hierarchy ergodic. However, additionally we have translated the independence events property of Bernoulli systems (see equation (76) ) into a quantum version in the sense of the expectation values (see equation (87)). The physical interpretation of this factorization is the quantum nocorrelation between the observables of a product of the type (87). This property was necessary to demonstrate the inclusion of quantum Bernoulli level within the quantum Kolmogorov level (see section 6.2).

We have just translated the four levels of the ergodic hierarchy to quantum language, and these levels from the lowest (ergodic) to the highest (Bernoulli) are schematized in the following diagram where the inclusions are strict.

\footnotetext{
${ }^{8}$ In the classical sense. In fact, when $\lambda>5$ the phase space of its classical analogue is chaotic with some surviving stable islands (see 6] pag. 10).

${ }^{9}$ Again, in the classical sense.
} 
In the next table we list the Quantum Ergodic Hierarchy levels in a compact way.

TABLE II: THE QUANTUM ERGODIC HIERARCHY (QEH)

LEVEL

Ergodic

Mixing

Kolmogorov

Bernoulli

\section{CONDITION EQUATION}

$$
\begin{aligned}
& \lim _{T \rightarrow \infty} \frac{1}{T} \int_{0}^{T}(\widehat{\rho}(t) \mid \widehat{O}) d t=\left(\widehat{\rho}_{*} \mid \widehat{O}\right) \text { (continuous) } \\
& \lim _{N \rightarrow \infty} \frac{1}{N} \sum_{k=0}^{N-1}(\widehat{\rho}(k) \mid \widehat{O}) d t=\left(\widehat{\rho}_{*} \mid \widehat{O}\right) \text { (discrete) }
\end{aligned}
$$

\section{PROPERTIES}

Cesaro limit equals to $\widehat{\rho}_{*}$

$\lim _{t \rightarrow \infty}(\widehat{\rho}(t) \mid \widehat{O})=\left(\widehat{\rho}_{*} \mid \widehat{O}\right)$ (continuous)

Weak limit equals to $\widehat{\rho}_{*}$

$\lim _{N \rightarrow \infty}(\widehat{\rho}(N) \mid \widehat{O})=\left(\widehat{\rho}_{*} \mid \widehat{O}\right)($ discrete $)$

$$
\begin{aligned}
& \lim _{n \rightarrow \infty}\left\{\left(\widehat{\rho}\left(n+m_{1}\right) \mid \widehat{O}_{1} \prod_{j=2}^{\infty} \widehat{O}_{j}\left(n+m_{j}\right)\right)\right. \\
& \left.-\prod_{j=2}^{\infty}\left(\widehat{\rho}\left(n+m_{1}\right) \mid \widehat{O}_{j}\left(n+m_{j}\right)\right)\left(\widehat{\rho}_{*} \mid \widehat{O}_{1}\right)\right\}=0
\end{aligned}
$$

Weak limit equals to $\widehat{\rho}_{*}$

$$
(\widehat{\rho}(t) \mid \widehat{O})=\left(\widehat{\rho}_{*} \mid \widehat{O}\right)
$$

In this table we can see the level of complexity of the condition that defines each level of the ergodic hierarchy. Starting at the lowest level, the ergodic, which translates into an average temporal of expectation values following by the mixing level corresponding to weak limit. And continuing with Kolmogorov level that represents a condition on a set of observables (the language translation of the sigma algebra) and ending with the Bernoulli level representing the null correlation for all time.

In section 7 we have presented two emblematic examples that show the relevance of the Quantum Ergodic Hierarchy: the Casati-Prosen model and the kicked rotator. We have explained their chaotic and decoherence behavior in terms of the QEH levels in a conceptual way.

For the Casati-Prosen model, the cancellation of the interference fringes can be deduced by the chaotic nature of its classical analogue in two ways, mixing and ergodic respectively: In the first case, for large times $t$ but shorter than $t^{*}$ (see section 7.1) the spectrum can be approximated by a quasi-continuous one and in the second case, otherwise we can maintain the spectrum discrete. But in both cases the equilibrium state $\widehat{\rho}_{*}$ expresses the type of decoherence of the initial Gaussian wavepacket $\widehat{\rho}(0)$, weak limit in the first case and Cèsaro limit in the second case.

For the kicked rotator we have characterized its quantum chaos transition, from small values of $\lambda$ to greater than 5, in terms of the Quantum Ergodic Hierarchy levels corresponding to each regime. This characterization is summarized in the next scheme. 


$$
\begin{gathered}
\lambda \ll 1 \Longrightarrow \text { integrable, regular behavior } \Longrightarrow \text { ergodic level } \\
\lambda \sim \lambda_{c}=0,9716 \ldots \Longrightarrow \text { stochastic and diffusive behavior } \Longrightarrow \text { ergodic level } \\
\lambda>5 \Longrightarrow \text { fully chaotic, exponential localization } \Longrightarrow \text { mixing and Bernoulli levels }
\end{gathered}
$$

We see that for the first two regimes, small values of $\lambda$ and values of $\lambda$ near to the critical value $\lambda_{c}=0,9716$ (see 6 page. 10 and 145), the regular and stochastic-diffusive behavior both correspond to the ergodic level of the QEH. Then QEH can not differentiate this two regimes. This is so because the Quantum Ergodic Hierarchy is a chaos classification for large times (in the limit $t \rightarrow \infty$ ) and therefore, we can not expect the chaos effects would occur in a finite interval of time.

Moreover, QEH allows to classify chaotic phenomena of large characteristic times as the decoherence of the Casati-Prosen model and the exponential localization of the kicked rotator for times $t \gg \tau l_{s}$. The exponential localization of the fully chaotic regime $\left(\lambda>5\right.$ and $\left.t \gg \tau l_{s}\right)$ of the kicked rotator corresponds to the Bernoulli level which is the most chaotic. On the positive side of The Quantum Ergodic Hierarchy we see that the regime less chaotic $\left(\lambda \lesssim \lambda_{c}\right)$ and more chaotic $(\lambda>5)$ correspond to the lowest and highest levels of QEH, ergodic and Bernoulli respectively. We consider that this agreement of QEH with other approximations to quantum chaos like the computational complexity in the Casati-Prosen model or the Floquet Theory in the kicked rotator are a positive first step of QEH as an alternative theoretical framework to study the phenomena of the quantum chaos.

Acknowledgements This paper was partially supported by the CONICET (Argentine Research Council) the FONCYT (the Argentine Fond for the Research) and the Buenos Aires University.

\section{References}

[1] J. Berkovitz, R. Frigg, F. Kronz, Stud. Hist. Phil. Mod. Phys., 37, 661-691, 2006.

[2] M. Castagnino, O. Lombardi, Phys. A, 388, 247-267, 2009.

[3] M. Hillery, M. O'Connell, R. Scully, E. Wigner, Phys. Rep., 106, 121-167, 1984.

[4] G. Casati, T. Prosen, Phys. Lett. A, 72, 032111, 2005.

[5] M. Castagnino, Phys. Lett. A, 357, 97-100, 2006.

[6] H. Stockmann, Quantum Chaos - An Introduction, Cambridge Univ. Press, Cambridge, 1999.

[7] G. Casati and B. Chirikov, Quantum Chaos: between order and disorder, Cambridge Univ. Press, Cambridge 1995.

[8] F. Haake, Quantum Signatures of Chaos, 2nd edition, Springer-Verlag, Heidelberg, 2001. 
[9] G. Belot, J. Earman, Stud. His. Philos. Mod. Phys., 28, 147-182, 1997.

[10] A. J. Lichtenberg, M. A. Lieberman, Regular and Chaotic Dynamics, Springer Verlag, New York, 1992.

[11] M. C. Gutzwiller, Chaos in Classical and Quantum Mechanics, Springer Verlag, New York, 1990.

[12] F. Benatti, Deterministic Chaos in Infinite Quantum Systems, Springer Verlag, Berlin, 1993.

[13] L. Ballentine, Quantum Mechanics - A Modern Development, World Scientific Publish Company, 1998.

[14] M. Berry, Physica Scripta, 40, 335-336, 1989.

[15] M. Castagnino, On the classical limit of quantum mechanics and the threat of fundamental graininess and chaos to the correspondence principle, submitted to Found. of Phys., 2009.

[16] R. Clifton and H. Halvorson, Phys. Rev. A, 61, 012108, 1999.

[17] R. Clifton, H. Halvorson and A. Kent, Phys. Rev. A, 61, 042101, 2000.

[18] A. Lasotta, M. Mackey, Probabilistic properties of deterministic systems, Cambridge Univ. Press, Cambridge, 1985.

[19] M. Mackey, Rev. Mod. Phys., 61, 981-1015, 1989.

[20] G. Dito, D. Sternheimer, arxiv math. QA/0201168, 2002.

[21] I. Cornfeld, S.V. Fomin, Y.G. Sinai, Ergodic theory, Springer, New York, 1982.

[22] M. Mackey, Time's arrow: the origin of thermodynamic behavior, Sringer Verlag, Berlin, 1992.

[23] K. Knuth, Neurocomputing, 67, 245-274, 2005.

[24] P. Bocchieri, A. Loinger, Phys. Rev. A, 107, 337-338, 1957.

[25] P. Bocchieri, A. Loinger, J. Phys. A, 4, 45, 2012.

[26] M. Castagnino, O. Lombardi, Philos. Sci., 72, 764-776, 2005. 